Pacific

Journal of

Mathematics

A SURPRISING BUBBLE CATASTROPHE

Henry C. Wente

Volume 189 No. 2

June 1999 


\title{
A SURPRISING BUBBLE CATASTROPHE
}

\author{
HenRy C. Wente
}

\begin{abstract}
Consider a tube with several orifices out of which fluid of a given volume protrudes. In gravity free conditions the equilibrium configuration will be one which minimizes total surface area subject to the volume constraint. The surface of each liquid drop will have the same constant mean curvature. Suppose that the orifices are cirlces with radii $r_{i}$ where each exposed drop is a spherical cap. We analyze this problem from the viewpoint of catastrophe theory. For a tube with two circular openings the interesting situation occurs when the configuration supports a double hemisphere $(h, h)$ equilibrium. This gives a cusp catastrophe with the radii $r_{1}, r_{2}$ as universal unfolding parameters. For the case of three openings with a triple hemisphere equilibrium $(h, h, h)$ we obtain an elliptic umbilic with the radii $r_{1}, r_{2}, r_{3}$ as unfolding parameters. Further surprising phenomena occur along the cusp lines emanating from the elliptic umbilic.
\end{abstract}

\section{Introduction.}

Consider a tube with two (widely separated) orifices whose bounding edges are circles of the same radius. Let a small amount of fluid be forced through the openings. In gravity-free conditions the equilibrium configuration will be one which minimizes the total surface area subject to a volume constraint. The controlling force is the surface tension of the liquid-air interface. In equilibrium each liquid drop will have the same constant mean curvature and so each drop will be a spherical cap of the same radius. For small volumes the unique minimizer will be two identical "small" caps which we identify as $(s, s)$. With increasing volume this situation persists until the two hemisphere state $(h, h)$ is reached. This is still a minimizing configuration. Beyond this critical volume there will exist three equilibrium configurations which we label as $(s, l),(l, l)$ and $(l, s)$. The two large cap configuration is unstable and the minimizers are either $(s, l)$ or $(l, s)$. This is a classic example of a pitch fork bifurcation and is often cited in texts as an example of a symmetry breaking phenomenon. See the book of A.W. Adamson [1] or the classic book on soap bubbles by C.V. Boys [3].

F. Duzaar and K. Steffen [6] considered the following generalization. Repeat the two bubble experiment but now assume we have a tube with three 
openings. Again for small volumes the unique minimizing configuration is three small drops $(s, s, s)$. As the volume is increased towards the critical state of three hemispheres the $(s, s, s)$ configuration remains a local minimizer of area and is stable. It turns out that the $(h, h, h)$ point is no longer a local minimizer of area for the volume constraint. The apparatus must jump to a distinct (and distant) new stable equilibrium state of the form $(s, s, l),(s, l, s)$ or $(l, s, s)$. A real catastrophe has occured.

As Duzaar and Steffen pointed out, there is a value $0<v^{*}<v_{c}$ where $v_{c}$ is the critical volume of three hemispheres such that the small bubble configuration is the area minimizer when $0<v<v^{*}$ while for $v^{*}<v<$ $v_{c}$ the small bubble configuration, although stable, is no longer a global minimizer. They exhibited this three bubble experiment as an example where the set of minimizing configurations for various volumes do not form a connected set. We note there is a jump in the mean curvature of the absolute minimizer as the volume passes through $v^{*}$.

The goal of this paper to analyze these experiments from the point of view of catastrophe theory. The two bubble pitchfork bifurcation is the hall mark of the cusp catastrophe. There will be two unfolding parameters which can be naturally produced by allowing the radii of the two circles $r_{1}, r_{2}$ to become parameters. For the three bubble apparatus a similar analysis will yield another one of R. Thom's seven elementary catastrophes, the elliptic umbilic. The universal unfolding now requires three parameters and again a suitable choice is the radii of the three circles $r_{1}, r_{2} r_{3}$.

In each experiment one wishes to eliminate the homothety as an expansion of this type produces a physically identical situation. This is most conveniently done by setting the total exposed volume $V_{T}=n(2 \pi / 3)$ where $n$ is the number of openings. The critical catastrophe then occurs when each $r_{i}=1$. One often likes to keep the openings fixed and allow the volume to increase from zero to infinity. This may be done by choosing a ray $\left(t r_{1}, t r_{2}, t r_{3}\right)$ emanating from the origin. As one moves from infinity to the origin along this ray one passes through a family of experiments equivalent (via a homothety) to that of increasing the volume with fixed openings.

For the three bubble experiment with the elliptic umbilic at $\left(r_{1}, r_{2}, r_{3}\right)=$ $(1,1,1)$ and $V_{T}=2 \pi$ there emanate three cusp lines which are part of the bifurcation set. One such cusp line lies in the plane $r_{1}=r_{2}$ and is tangent to the ray $r_{1}=r_{2}=r_{3}$ at $(1,1,1)$. This cusp line has two parts. One part consists of points $r_{1}=r_{2}>r_{3}$ which support equilibrium configurations ( $h, h, s)$ of total volume $2 \pi$ while the other piece will support configurations of the form $(h, h, l)$ of total volume $2 \pi$. We refer to the first case as branch $[A]$ and the second as branch $[B]$.

On branch $[A]$ all points but one determine a cusp catastrophe. It turns out that as one passes through this critical point $P^{*}=\left(r_{1}^{*}, r_{2}^{*}, r_{3}^{*}\right)$ with $r_{3}^{*}<r_{1}^{*}=r_{2}^{*}$ and $r_{3}^{*} / r_{1}^{*} \cong .95$ along the cusp curve there is a reversal in the 
direction of bifurcation. There is a switch from the cusp to its dual. The critical point $P^{*}$ is a catastrophe of order six, the butterfly. This catastrophe requires four parameters for a universal unfolding. The three we have are not sufficient. We present two different perturbations of the system either of which will generate the necessary fourth unfolding parameter. One is to introduce a gravitational potential on the exposed mass of the fluid leading to new equilibrium configurations in the form of pendant and sessile drops. The other is to introduce surface tension as a parameter. The energy then becomes a weighted sum of the areas of the individual drops. A surprise (to me at least) is that a perturbation of the boundary curves away from circles fails to produce a universal unfolding at the butterfly point.

In Section 2 we derive the basic formulas needed in our analysis and prove some elementary but useful lemmas. As essential point is that we can reduce the problem to a finite dimensional setting by the device of replacing any oriented surface spanning a circle by a spherical cap spanning the same circle, enclosing the same volume but having smaller area. We also give a simple convincing argument why there is a real catastrophe in the three bubble case. In Section 3 we give a quick review of the key definitions and tools of catastrophe theory we shall need. Our main source is the book of D. Castrigiano and S. Hayes [5] and the exposition of T. Poston and I. Stewart [10]. In Section 4 we carry out the analysis for the two bubble experiment for the sake of completeness and to prepare us for the three bubble case. In Section 5 we analyze the three bubble configuration. First we display the elliptic umbilic at the three hemisphere state and show that the three parameters $r_{1}, r_{2}, r_{3}$ provide a universal unfolding. We then demonstrate the existence of the butterfly catastrophe on branch $[A]$ of the cusp line, the branch with $(h, h, s)$ equilibria. In the final Section 6 we produce the universal unfolding for the butterfly. We show that either the introduction of a gravitational potential or a perturbation of the surface tension succeeds while any attempt to produce a universal unfolding by bending the boundary circles fails.

There have been many applications of Catastrophe Theory to problems in the physical sciences and geometry. Of special relevance is the paper of M. Beeson and A. Tromba [2]. They study Enneper's Minimal Surface as a solution to Plateau's Problem spanning a family of Jordan curves $\Gamma_{r}$ lying in the surface and increasing in size. Initially, Enneper's surface is the only solution, being the area minimizer. At a certain value $r=1$ the Enneper's surface losses stability and there is a bifurcation into three minimal surfaces spanning $\Gamma_{r}$ when $r>1$. This phenomenon was first observed by J.C.C. Nitsche $[\mathbf{9}]$. Beeson and Tromba carried out the detailed analysis, identified the cusp catstrophe and produced unfolding. An extension of this approach with other examples was recently carried out by J. Büch [4]. A further interesting application of Catastrophe Theory in the study of minimal surfaces 
is described by A.T. Fomenko in [8]. In his book a swallowtail catastrophe is exhibited where the unfolding parameters correspond to bending of the boundary curve.

The author wishes to express a special thanks to the referee who made a number of very pertinent and beneficial suggestions.

\section{Essential Formulas and Observations.}

First we display the formulas for the surface area and volume of a single spherical cap bounded by a circle of radius $r$. Let $\mathcal{C}$ be the circular arc in the upper half plane whose center is $(0, t)$ and which passes through $(r, 0)$. The surface is generated by rotating this arc about the $y$-axis while the point $(r, 0)$ generates the bounding circle. We shall use $t$ as a convenient parameter. One has

$$
\begin{aligned}
A(r, t) & =2 \pi\left(r^{2}+t^{2}+t \sqrt{r^{2}+t^{2}}\right) \\
V(r, t) & =\frac{\pi}{3}\left[2\left(r^{2}+t^{2}\right)^{\frac{3}{2}}+3 t\left(r^{2}+t^{2}\right)-t^{3}\right] .
\end{aligned}
$$

Note that for $t<0$ we have a small cap, for $t=0$ the hemisphere and for positive $t$ the large spherical cap. One checks that $d A / d V=2 / a=2 H$ where $H$ is the mean curvature and $a=\sqrt{r^{2}+t^{2}}$. In particular we will use these formulas when $r=1$.

$$
\begin{aligned}
& a(t)=A(1, t)=2 \pi\left(1+t^{2}+t \sqrt{1+t^{2}}\right) \\
& v(t)=V(1, t)=\frac{\pi}{3}\left[2\left(1+t^{2}\right)^{\frac{3}{2}}+3 t\left(1+t^{2}\right)-t^{3}\right] .
\end{aligned}
$$

One finds that

$$
\dot{v}(t)=\pi\left(t+\sqrt{1+t^{2}}\right)^{2}
$$

is positive so that we have $a=a(v)$.

Lemma 2.1. The area of a spherical cap as a function of volume has the following derivatives

$$
a^{\prime}(v)=2 / \sqrt{1+t^{2}}
$$

$$
a^{\prime \prime}(v)=(-2 / \pi)\left[t /\left(1+t^{2}\right)^{\frac{3}{2}}\right]\left[\sqrt{1+t^{2}}-t\right]^{2}
$$

$$
a^{\prime \prime \prime}(v)=\left(-2 / \pi^{2}\right)\left[\left(\sqrt{1+t^{2}}-t\right)^{4} /\left(1+t^{2}\right)^{\frac{5}{2}}\right]\left[1-2 t^{2}-2 t \sqrt{1+t^{2}}\right]
$$

$a^{(4)}(v)=\left(2 / \pi^{3}\right)\left[\left(\sqrt{1+t^{2}}-t\right)^{6} /\left(1+t^{2}\right)^{\frac{7}{2}}\right]\left[6+t-14 t^{2}\left(\sqrt{1+t^{2}}+t\right)\right]$.

For $t=0$ we have $v=v_{c}=2 \pi / 3$. We find 
(a) $a(v)$ has an inflection point at $v=v_{c}$ with $a^{\prime \prime}\left(v_{c}\right)=0, a^{\prime \prime}(v)$ is positive for $0 \leq v<v_{c}$ and $a^{\prime \prime}(v)$ is negative for $v>v_{c}$. Observe that $a^{\prime \prime}(0)=$ $8 / \pi$.

(b) $a^{\prime \prime \prime}(v)=0$ when $t=\sqrt{2} / 4$ which corresponds to a value $v_{1}>v_{c}$. We have $a^{\prime \prime \prime}(v)$ negative for $0<v<v_{1}$ and positive for $v>v_{1}$. Furthermore $a^{\prime \prime \prime}\left(v_{c}\right)=-2 / \pi^{2}$.

(c) $a^{(4)}(v)$ is positive in some interval about $v=v_{c}$ with $a^{(4)}\left(v_{c}\right)=12 / \pi^{3}$. It is negative outside this interval.

It is very convenient to rescale this function by setting $v=(2 \pi / 3) u$ and $E=a / \pi$.

Definition 2.1. The rescaled energy function is $E=F(u)=\frac{1}{\pi} a\left(\frac{2 \pi}{3} u\right)$ where $a(v)$ is given by $(2.2)$.

Corollary. The rescaled function $E=F(u)$ has the following properties, (see Figure 2).

a) $F(u)$ is an increasing function of $u$ with $F^{\prime}(u)$ positive for $u>0$. We have $F(0)=1, F^{\prime}(0)=0, F(1)=2$ and $F^{\prime}(1)=4 / 3$.

b) $F(u)$ has an inflection point at $u=1$ with $F^{\prime \prime}(1)=0 . F^{\prime \prime}(u)$ is positive for $0<u<1$ and negative for $u>1, F^{\prime \prime}(0)=32 / 9$.

c) $F^{\prime \prime \prime}(u)=0$ at a value $u_{1}>1 . F^{\prime \prime \prime}(u)$ is negative for $0<u<u_{1}$ and positive for $u>u_{1}$ with $F^{\prime \prime \prime}(0)=0$ and $F^{\prime \prime \prime}(1) \equiv-\gamma=-16 / 27$.

d) $F^{(4)}(u)$ is positive in some interval about $u=1$ and is negative outside that interval with $F^{(4)}(1) \equiv \delta=64 / 27$.

Definition 2.2 (The Experiment). Consider the apparatus consisting of a tube with $n$ circular orifices of corresponding radii $r_{i}$. Let the total exposed volume be $V_{T}=(2 \pi / 3) U_{T}$ where generally $U_{T}=n$. By replacing each bubble by its equivalent spherical cap, our state space $S$ consists of all $n$ tuples $\bar{u}=\left(u_{1}, u_{2}, \ldots, u_{n}\right)$ with $u_{1}+u_{2}+\cdots+u_{n}=n$, where we assume each $u_{i} \geq 0$. The energy is given by

$$
E=\sum_{i=1}^{n} r_{i}^{2} F\left(u_{i} / r_{i}^{3}\right)
$$

where $E=($ area $) / \pi$. We look for equilibria of this function subject to the constraint $u_{1}+u_{2}+\cdots+u_{n}=U_{T}=n$.

Using the constraint condition and regarding $E$ as a function of $\left(u_{1}, \ldots\right.$, $u_{n-1}$ ) one finds that at an equilibrium point there is a constant $H$ with

$$
\frac{1}{r_{i}} F^{\prime}\left(u_{i} / r_{i}^{3}\right)=2 H \quad i=1,2, \ldots, n .
$$

Each spherical cap has the same mean curvature. We shall label any spherical cap by $s$ (small), $h$ (hemisphere) or $l$ (large). We have the following result for equilibrium configurations consisting of small caps. 
Lemma 2.2. Let $r_{i}>0$ be given and let $S^{\prime}$ be the set of states $\bar{u}=$ $\left(u_{1}, \ldots, u_{n}\right)$ where $u_{i} \leq r_{i}^{3}$ so that each bubble is a small (possibly hemispherical ) cap. Let $\bar{u}^{*}=\left(u_{1}^{*}, u_{2}^{*}, \ldots, u_{n}^{*}\right)$ be an equilibrium configuration for $E$ subject to the constraint $u_{1}+u_{2}+\cdots+u_{n}=n$ and which lies in $S^{\prime}$.

a) The stae $\bar{u}^{*}$ is a minimizer of $E$ in the state space $S^{\prime}$ subject to $u_{1}+$ $u_{2}+\cdots+u_{n}=n$.

b) If $\bar{u}^{*}$ is an interior point of $S^{\prime}$ then $\bar{u}^{*}$ is a strictly stable local minimizer of $E$ in $S$ as well.

Proof. By the Corollary to Lemma 2.1 we see that the function $E$ given by $(2.4)$ is a strictly convex function of $\left(u_{1}, u_{2}, \ldots, u_{n}\right)$ on the set $S^{\prime}$. Its Hessian is diagonal with entries

$$
E_{u_{i} u_{i}}=\frac{1}{r_{i}^{4}} F^{\prime \prime}\left(u_{i} / r_{i}^{3}\right) \geq 0 .
$$

It follows that on the linear convex set $u_{1}+u_{2}+\cdots+u_{n}=n$ the function $E$ has a unique minimizing point $\bar{u}^{*}$. If this minimum is at an interior point then it must be stable as the Hessian is positive definite. Also, if $\bar{u}^{*}$ is an interior equilibrium point then by the convexity of $E$ it will be a stable minimizer for the constrained problem.

Theorem 2.1. We collect the following facts.

(a) Any equilibrium with two or more large caps is unstable.

(b) Suppose $n=2$ and fix $r_{1}=r_{2}$. As we allow the total volume to vary there are two smooth families of equilibria. One is the symmetric family $(s, s) \rightarrow(h, h) \rightarrow(l, l)$, the other is $(s, l) \rightarrow(h, h) \rightarrow(l, s)$ giving the pitchfork bifurcation at $(h, h)$. The stable minimizers are $(s, s),(h, h)$ and $(s, l),(l, s)$ while $(l, l)$ is unstable.

(c) Suppose $n=2$ and $r_{1}>r_{2}$. There are now two smooth disjoint families. One is $(s, s) \rightarrow(l, s)$ while the other is $(s, l) \rightarrow(l, l)$. We can parametrize either family by $t$, the center of the first drop. (One could also use the volume $u_{1}$ of the first drop as parameter.) On the first family the rate of change of volume $\dot{V}(t)$ is positive. All configurations are stable equilibria and are minimizers for the constrained problem. On the second family the volume function $V(t)$ is a convex function with limit $V(t)=+\infty$ at each end. The configurations $(s, l)$ for $t$ near $-\infty$ are initially stable. There is a point before the $(h, l)$ configuration is reached where $\dot{V}(t)=0$ giving a loss of stability. The $(h, l)$ state is not stable. The point $t^{*}$ where $\dot{V}\left(t^{*}\right)=0$ with state $\left(s^{*}, l^{*}\right)$ corresponds to a fold catastrophe. In particular there are states $(s, l)$ which are unstable.

Proof. To prove (a) it is sufficient to consider the case of two bubbles. Suppose $(l, l)$ is an equilibrium configuration with $u_{1}+u_{2}=2$ with the energy 
function $E$ given by (2.4). Set $u=u_{1}$, so that $u_{2}=2-u$. In equilibrium we have $E_{u}=0$ so that condition (2.5) is satisfied. But then

$$
E_{u u}=\frac{1}{r_{1}^{4}} F^{\prime \prime}\left(u_{1} / r_{1}^{3}\right)+\frac{1}{r_{2}^{4}} F^{\prime \prime}\left(u_{2} / r_{2}^{3}\right) .
$$

For large caps $u_{1} / r_{1}^{3}>1$ and $u_{2} / r_{2}^{3}>1$ and so $E_{u u}$ is negative by the properties of $F(u)$ given in the Corollary to Lemma 2.1.

Statement (b) follows from (a) and the fact that the constrained problem always has a minimizer.

For the families in (c) the volume $V_{1}(t)$ of the first bubble is given by (2.1). For the first family the second bubble is a small cap whose radius $a=\sqrt{r_{1}^{2}+t^{2}}$ is that of the first bubble. The volume $V_{2}$ of the small cap is given by $V_{2}=(\pi / 6) x\left(x^{2}+3 r_{2}^{2}\right)$ where $x=a-\sqrt{a^{2}-r_{2}^{2}}$ is the height of the cap. One finds

$$
\dot{V}(t)=\pi\left\{(a+t)^{2}-\frac{t\left(a-\sqrt{a^{2}-r_{2}^{2}}\right)}{\sqrt{a^{2}-r_{2}^{2}}}\right\} .
$$

Clearly this is positive when $t \leq 0$. For positive $t$ one checks that the expression on the right is decreased if we replace $r_{2}$ by $r_{1}$. This new expression simplifies to $4 \pi a t$ and so $\dot{V}(t)>0$ for all $t$.

Note. It is easily seen from the isoperimetric inequality that when $r_{1}>r_{2}$ the unique minimizing configuration is obtained by setting the second bubble to be a small cap. Let $\left(c_{1}, c_{2}\right)$ be a minimizing configuration. $c_{1}$ is a large or small cap of some sphere bounded by a circle of radius $r_{1}$. Since $r_{2}<r_{1}$ there is room to fit the small cap $c_{2}$ on that remaining cap of the sphere not covered by $c_{1}$. The assertion follows.

In the same manner for the second family where $c_{2}$ is a large cap we have the same formula for $V_{1}(t)$ but $V_{2}(t)$ is now the volume of the large cap determined by $t$. One finds

$$
\begin{aligned}
\frac{d^{2}\left(V_{1}+V_{2}\right)}{d t^{2}}= & \left(a+\sqrt{a^{2}-r_{2}^{2}}\right)\left(\frac{t}{a}+\frac{t}{\sqrt{a^{2}-r_{2}^{2}}}\right)^{2} \\
& +\frac{\pi}{2}\left(2 a^{2}+2 a \sqrt{a^{2}-r_{2}^{2}}\right)\left(\frac{r_{1}^{2}}{a^{3}}+\frac{r_{1}^{2}-r_{2}^{2}}{\left(a^{2}-r_{2}^{2}\right)^{\frac{3}{2}}}\right)
\end{aligned}
$$

which is positive for all $t$. One sees that when $t=0$ where the first cap is a hemisphere that $\dot{V}_{1}(0)$ is positive while the second drop has a turn around in curvature showing that $\dot{V}_{2}(0)=0$. Therefore on this second family the total volume $V(t)$ reaches a minimum at some point $t^{*}<0$ when the configuration is in an $(s, l)$ mode. We now check loss of stability at the turn around point $t^{*}$. Let us set the first volume $u_{1}=u$ as parameter with $u_{1}+u_{2}=U(u)$ where 
$U(u)$ is the scaled total volume. This scaled volume $U(u)$ is determined by the equilibrium condition (2.5). The stability is determined by the sign of $E_{u u}$ given by (2.7). We have in equilibrium

$$
\frac{1}{r_{1}} F^{\prime}\left(u / r_{1}^{3}\right)-\frac{1}{r_{2}} F^{\prime}\left([U(u)-u] / r_{2}^{3}\right)=0 .
$$

Differentiating with respect to $u=u_{1}$ and rearranging we find

$$
\frac{1}{r_{2}^{4}} F^{\prime \prime}\left(u_{2} / r_{2}^{3}\right) \cdot[d U / d u]=\frac{1}{r_{1}^{4}} F^{\prime \prime}\left(u_{1} / r_{1}^{3}\right)+\frac{1}{r_{2}^{4}} F^{\prime \prime}\left(u_{2} / r_{2}^{3}\right) .
$$

Since $c_{2}$ is a large cap we have $u_{2} / r_{2}^{3}>1$ and $F^{\prime \prime}\left(u_{2} / r_{2}^{3}\right)<0$. The right side of our equation is opposite the sign of $(d U / d u)$ which is essentially $d V / d t$ and assertion (c) follows.

We conclude this section by discussing one aspect of the three bubble problem. Suppose $r_{1}=r_{2}=r_{3}$ and consider the families which pass through the three hemisphere $(h, h, h)$ state. The symmteric family is $(s, s, s) \rightarrow$ $(h, h, h) \rightarrow(l, l, l)$. This is a family of increasing volume where the $(s, s, s)$ segment is stable while the $(l, l, l)$ segment is not, and for small exposed volume the $(s, s, s)$ family will be a global minimizer. The three other families are permutations of each other, for example $(s, s, l) \rightarrow(l, l, s)$. Note that this family is equivalent to a two bubble picture where $r_{2}=0$ and the second bubble is a complete sphere, (simply put the spherical caps two and three together to form an entire sphere). One can then use (2.9) to see that the total volume of this family is a convex function. As earlier, one observes that the total volume $V(t)$ obtains its minimum at some point before the three hemisphere state is reached. This family becomes unstable when $V_{\text {min }}$ is obtained. Therefore in a neighbourhood of $(h, h, h)$ the only stable configurations are three small caps. A jump in the equilibrium must occur as the volume is increased beyond the three hemisphere state. We shall see in Section 5 that even $(h, h, h)$ is not a local minimizer.

\section{Review of Catastrophe Theory.}

We now give a quick summary of those definitions and results of catastrophe theory which will be needed. We follow the discussion in the book of Castrigiano and Hayes [5].

Definition 3.1. Let $\mathcal{E}$ be the algebra of germs of $C^{\infty}$-functions defined in a neghbourhood of $x=0$ in $\mathbb{R}^{n}$. Two functions $f$ and $g$ determine the same germ if they agree on some neighbourhood of the origin. The germ determined by $f$ is denoted by $[f]$.

Definition 3.2. $m \subset \mathcal{E}$ is the (maximal) ideal of germs of functions which vanish at $x=0$. Given $m$ one can also form the ideals $m^{k}$ where

$$
m^{k}=\left\{f\left|D^{\alpha} f(0), \quad\right| \alpha \mid<k\right\}, \quad k \geq 1 .
$$


Definition 3.3. Let $\mathcal{A}$ be the set of all local $C^{\infty}$-diffeomorphisms $\psi$ of a neighbourhood of $x=0$ with $\psi(0)=0$.

Note: $D \psi(0)$ is invertible. Frequently we will have $D \psi(0)=$ identity.

Definition 3.4. If $A \subset \mathcal{E}$ is a subset of $\mathcal{E}$ then $\langle A\rangle$ is the linear subspace of $\mathcal{E}$ generated by $A$ while $\langle A\rangle_{\mathcal{E}}=\langle\mathcal{E} A\rangle$ is ideal generated by $A$.

Definition 3.5. Let $f \in \mathcal{E}$. The $k$-jet of $f$ about $x=0$ is the polynomial of degree $k$

$$
\begin{gathered}
j^{k}(f)=\sum_{|\alpha| \leq k} \frac{1}{\alpha !} D^{\alpha} f(0) x^{\alpha} \\
\alpha=\left\langle\alpha_{1}, \ldots, \alpha_{n}\right\rangle, x^{\alpha}=x_{1}^{\alpha_{1}} \cdots x_{n}^{\alpha_{n}}, \alpha !=\alpha_{1} ! \cdots \alpha_{n} !
\end{gathered}
$$

Note that $J^{k}$, the space of $k$-jets in $\mathcal{E}$ is isomorphic to $\mathcal{E} / m^{k+1}$.

Definition 3.6. $f$ is $k$-determinant at $x=0$ if given $g \in \mathcal{E}$ with $j^{k}(f)=$ $j^{k}(g)$ there exists a local diffeomorphism $\psi \in \mathcal{A}$ with $g=f \cdot \psi$. The smallest integer $k$ such that $f$ is $k$-determined is called the determinancy of $f$, denoted by $\operatorname{det}[f]$.

We will display R. Thom's short list at the end of this section but we now note that

(1) $f(x) \equiv c$ is not finitely determined.

(2) $f(x)$ is $k$-determined $\Rightarrow f(x)+c$ is also.

(3) $\nabla f(0) \neq 0$ implies $\operatorname{det}[f]=1$.

(4) $\nabla f(0)=0, D^{2} f(0)$ nondegenerate $\Rightarrow \operatorname{det}[f]=2$.

Definition 3.7. The Jacobi Ideal $\mathcal{J}(f)$ is the ideal in $\mathcal{E}$ generated by the first partial derivatives $f_{i}(x)=\partial f / \partial x_{i}, i=1,2, \ldots, n$.

$$
\mathcal{J}(f)=\left\langle f_{1}(x), f_{2}(x), \ldots, f_{n}(x)\right\rangle_{\mathcal{E}} .
$$

Theorem 3.1 (Test for determinancy). Let $f \in \mathcal{E}$ and form $\mathcal{J}(f)$.

(1) If $m^{k+1} \subset\left\langle m^{2} \mathcal{J}(f)\right\rangle$ then $f$ is $k$-determined.

(2) $f$ is finitely determined if there exists $k$ with $m^{k} \subset\langle m \mathcal{J}(f)\rangle$. If $k$ is the smallest integer for which this is true then $\operatorname{det}[f]=k$ or $k-1$.

(3) If $f$ is $k$-determined, then $m^{k+1} \subset\langle m \mathcal{J}(f)\rangle$.

Definition 3.8. Let $f \in m^{2}$ be a finitely determined germ with $\operatorname{det}[f]>2$. For such $f$

$$
\text { codimension }[f] \equiv \operatorname{cod}[f]=\operatorname{dim}(m / \mathcal{J}(f)) \text {. }
$$

Definition 3.9. An unfolding of a germ $f$ is a local $C^{\infty}$ map $F: \mathbb{R}^{n+L} \rightarrow$ $\mathbb{R}$ with $F=F(x, r)$ where $x=\left(x_{1}, \ldots, x_{n}\right), r=\left(r_{1}, \ldots, r_{L}\right)$ such that $F(0, r)=0$ and $F(x, 0)=f(x)$.

Definition 3.10. An unfolding $G(y, s)$ is induced by $F(x, r)$ if there exist smooth functions 
(a) $x=\varphi(y, s)$ with $y=\varphi(y, 0)$,

(b) $r=\psi(s)$ with $\psi(0)=0$,

(c) $\gamma(s) \in \mathbb{R}$ with $\gamma(0)=0$,

such that $G(y, s)=F(\varphi(y, s), \psi(s))+\gamma(s)$.

Definition 3.11. An unfolding $F$ is versal if any other unfolding $G$ is induced by $F$. It is universal if it is versal and the number of parameters $L$ is minimal and equal cod $[f]$.

Theorem 3.2. Let $f$ be finitely determined with $\operatorname{cod}(f)=L$. Let $g_{1}(x)$, $g_{2}(x), \ldots, g_{L}(x)$ span the linear space $[m / \mathcal{J}[f]]$. Then

$$
F(x, r)=f(x)+\sum_{i=1}^{L} r_{i} g_{i}(x)
$$

is a universal unfolding of $f$.

R. Thom's Seven Elementary Catastrophes \& Universal Unfoldings.

\begin{tabular}{|c|c|c|c|}
\hline Germ \& Unfolding & $\operatorname{det}[f]$ & $\operatorname{cod}[f]$ & Name \\
\hline $\pm x^{3}-a x$ & 3 & 1 & fold \\
\hline $\pm x^{4}-a x^{2}-b x$ & 4 & 2 & cusp \\
\hline$x^{5}-a x^{3}-b x^{2}-c x$ & 5 & 3 & swallow tail \\
\hline $\pm x^{6}-a x^{4}-b x^{3}-c x^{2}-d x$ & 6 & 4 & butterfly \\
\hline$\left(x^{3}-x y^{2}\right)-a\left(x^{2}+y^{2}\right)$ & 3 & 3 & elliptic umbilic \\
$-b x-c y$ & & & \\
\hline$\left(x^{3}+y^{3}\right)-a x y-b x-c y$ & 3 & 3 & hyperbolic umbilic \\
\hline$\left(x^{2} y+y^{4}\right)-a x^{2}-b y^{2}$ & 4 & 4 & parabolic umbilic \\
$-c x-d y$ & & & \\
\hline
\end{tabular}

Theorem 3.3 (Splitting Lemma). Let $f \in m^{2}$ and suppose the hessian $D^{2} f(0)$ has rank $L<n$. There exists a local diffeomorphism about $x=0$ so that in the new coordinates $f$ takes the form

$$
f(y)=\sum_{i=1}^{L} \epsilon_{i} y_{i}^{2}+g\left(y_{L+1}, \ldots, y_{n}\right)
$$

where $\epsilon_{i}= \pm 1$ and $g \in m^{3}$.

The following theorem allows us to identify universal unfoldings. 
Theorem 3.4. Let $F(x, r)$ with $x \in \mathbb{R}^{n}, r \in \mathbb{R}^{L}$ be an unfolding of the finitely determined function $f(x)$ about $(x, r)=(0,0)$. Let

$$
g_{i}(x)=F_{r_{i}}(x, 0)-F_{r_{i}}(0,0) \in m
$$

and let $V(F)$ be the linear space generated by $g_{1}(x), \ldots, g_{L}(x) . F(x, r)$ is a versal unfolding if the image of $V(F)$ under the quotient map equals $[m / \mathcal{J}(f)]$. It is universal if in addition $L=\operatorname{cod}[f]=\operatorname{dim}(m / \mathcal{J}(f))$. Furthermore the unfolding

$$
G(x, r)=f(x)=\sum_{i=1}^{r} r_{i} g_{i}(x)
$$

is then also universal.

Finally we define the following sets.

Definition 3.12. Let $F(x, r)$ be a universal unfolding of the finitely determined germ $f$.

(1) The catastrophe surface $M_{F} \subset \mathbb{R}^{n+L}$ is the set $M_{F}=\left\{(x, r) \mid D_{x} F(x, r)\right.$ $=0\}$.

(2) The catastrophe set $C_{F}$ is the subset of $M_{F}$ consisting of those points $(x, r)$ in $M_{F}$ for which $D_{F}^{2}$ is degenerate.

(3) The bifurcation set $B_{F}$ is the projection of $C_{F}$ onto the parameter space $\mathbb{R}^{L}$.

In the next section we shall encounter the function $E(x)$ where $D E\left(x_{0}\right)=$ $0, D^{2} E\left(x_{0}\right)$ is degenerate and $E(x)$ is finitely determined. We can extend the definition of unfolding as given in Definition 3.9 by setting $F(w)=$ $E\left(x_{0}+w\right)-E\left(x_{0}\right)$ so that $F(0)=0$ and the degenerate point is at $w=0$.

\section{The Double Pipe Catastrophe.}

For the double pipe with openings $r_{1}, r_{2}$ and normalized volumes $u_{1}, u_{2}$ the energy function $E\left(u, r_{1}, r_{2}\right)$ is given by (2.4) where we set $u=u_{1}$ and $u_{1}+$ $u_{2}=2$. The double hemisphere occurs when $r_{1}=r_{2}=1$. The catastrophe surface $M_{E}$ is the set of points $\left(u, r_{1}, r_{2}\right)$ in $\mathbb{R}^{3}$ where

$$
E_{u}=\frac{1}{r_{1}} F^{\prime}\left(u_{1} / r_{1}^{3}\right)-\frac{1}{r_{2}} F^{\prime}\left(u_{2} / r_{2}^{3}\right)=0 .
$$

The catastrophe set $C_{E}$ are those points in $M_{E}$ for which $E_{u u}=0$.

$$
E_{u u}=\frac{1}{r_{1}^{4}} F^{\prime \prime}\left(u_{1} / r_{1}^{3}\right)+\frac{1}{r_{2}^{4}} F^{\prime \prime}\left(u_{2} / r_{2}^{3}\right)=0 .
$$

Its projection onto the parameter space $\left(r_{1}, r_{2}\right)$ is the bifurcation set $B_{E}$. The cusp will occur when $\left(u, r_{1}, r_{2}\right)=(1,1,1) \in C_{E}$ and $(1,1) \in B_{E}$. At 
$(1,1,1)$ we see that $E_{u u}=E_{u u u}=0$ while

$$
D_{u}^{4}=\frac{1}{r_{1}^{10}} F^{(4)}\left(u_{1} / r_{1}^{3}\right)+\frac{1}{r_{2}^{10}} F^{(4)}\left(u_{2} / r_{2}^{3}\right)
$$

and so $D_{u}^{4} E(1,1,1)=2 F^{(4)}(1)=2 \delta=128 / 27$ using the Corollary to Lemma 2.1. Thus

$$
j^{4}(E)=\frac{2 \delta}{24}(u-1)^{4}=(16 / 81)(u-1)^{4}
$$

or if we set $x=u-1$, then $j^{4}(E)=(16 / 81) x^{4}$. The determinacy of $E$ at $(1,1,1)$ is four, giving us the cusp catastrophe.

We now show that the parameters $r_{1}, r_{2}$ are unfolding parameters and that this unfolding is universal. We know that $\operatorname{cod}[E]=2$ where $m \bmod \mathcal{J}(E)$ is spanned by $\left\{(u-1),(u-1)^{2}\right\}$. We now calculate

$$
T_{i}(u-1)=E_{r_{i}}(u, 1,1)-E_{r_{i}}(1,1,1) \quad i=1,2
$$

and expanded these in power of $(u-1)=x$. We find

$$
\begin{aligned}
& T_{1}(x)=(-4 / 3) x+(8 / 9) x^{2}+\cdots \\
& T_{2}(x)=(4 / 3) x+(8 / 9) x^{2}+\cdots
\end{aligned}
$$

using the properties of $F(u)$ given in the Corollary to Lemma 2.1. Clearly the pair $T_{1}, T_{2}$ does span $m \bmod \mathcal{J}(E)$ and we have a universal unfolding by Theorem 3.4. It is equivalent to the unfolding

$$
E\left(u, r_{1}, r_{2}\right) \cong(16 / 81) x^{4}+r_{1} T_{1}(x)+r_{2} T_{2}(x) .
$$

More symmetric parameters are obtained by setting $s_{1}=r_{1}+r_{2}, s_{2}=r_{1}-r_{2}$. With these parameters the unfolding becomes

$$
E\left(u, s_{1}, s_{2}\right) \cong(16 / 81) x^{4}+\left(8 s_{1} / 9\right) x^{2}-\left(4 s_{2} / 3\right) x .
$$

This is the standard unfolding of the cusp as given in the table. The tangent direction through the cusp is the ray from the origin through $(1,1)$.

To complete the bifurcation set one must include the fold lines. These are two symmetric curves with a cusp at $(1,1)$. One branch goes to the $r_{1}$ axis and the other to the $r_{2}$ axis.

Fix $r_{1}>r_{2}>0$. As discussed in Section 2 there are two families of equilibria namely $(A):(s, s) \rightarrow(l, s)$ and $(B):(s, l) \rightarrow(l, l)$. By Theorem 2.1 the volume function on family $(A)$ is strictly monotonic, $\dot{V}(t)>0$, and this family provides the minimizers. On Family $(B)$ the volume function is strictly convex and there is a unique point $t^{*}<0$ with $\dot{V}\left(t^{*}\right)=0$. This determines the fold point on a given ray $r_{2} / r_{1}=$ constant.

When $r_{2}=0$ we are in the problem of a spherical cap plus an entire sphere. For such a configuration we have $V(t)=V_{1}(t)+V_{2}(t)$ where $V_{1}(t)$ is the volume of the cap $(2.1)$ and $V_{2}(t)$ is the volume of an entire sphere 
of radius $a$ where $a^{2}=r_{1}^{2}+t^{2}$. If $r_{1}=1$ then $\dot{V}\left(t^{*}\right)=0$ when $t^{*} \cong-.1$, $V\left(t^{*}\right) \cong(4 \pi / 3)(1.447)$.

For our normalization the total volume $V_{T}=4 \pi / 3$ giving us $r_{1}^{*} \cong$ $(1.447)^{-1 / 3} \cong .884$ [see Figure 3$]$.

\section{The Triple Pipe Catastrophe.}

We now consider the triple pipe configuration with bounding circles of radii $r_{1}, r_{2}, r_{3}$. The energy function is given by (2.4) where the exposed volume is $v_{i}=(2 \pi / 3) u_{i}, u_{1}+u_{2}+u_{3}=3$

$$
E\left(u_{1}, u_{2}, r_{1}, r_{2}, r_{3}\right)=\sum_{i=1}^{3} r_{i}^{2} F\left(u_{i} / r_{i}^{3}\right), \quad u_{1}+u_{2}+u_{3}=3 .
$$

We describe the catastrophe at the triple hemisphere point $(h, h, h)$ where $r_{1}=r_{2}=r_{3}=1$.

Theorem 5.1. The point $(\bar{u}, \bar{r})=(1,1,1,1,1) \in \mathbb{R}^{5}$ lies on the catastrophe set $C_{E}$ of the energy function $E$. At this point $E$ is totally degenerate with $D_{u} E=D_{u}^{2} E=0$. The energy is a finitely determined function with $\operatorname{det}[E]=$ 3 giving us an elliptic umbilic point as listed in the table. This catastrophe has codimension three and the parameters $\left(r_{1}, r_{2}, r_{3}\right)$ provide a universal unfolding. The bifurcation set for the elliptic umbilic contains three cusp lines which are tangent at $(1,1,1)$ to the ray based at the origin.

Proof. As we are in equilibrium we know that $D_{u} E=0$. Furthermore $D_{u}^{2} E=$ 0 as well since $F^{\prime \prime}(1)=0$. The Hessian is totally degenerate and we consider the third derivatives. A direct computation using the properties of $F(u)$ as listed in the Corollary to Lemma 2.1 gives

$$
j^{3}(E)=(\gamma / 2)\left[\left(u_{1}-1\right)^{2}\left(u_{2}-1\right)+\left(u_{1}-1\right)\left(u_{2}-1\right)^{2}\right]
$$

where $F^{\prime \prime \prime}(1)=-\gamma=-16 / 27$. Set $u_{1}=x+1, u_{2}=y+1$ so then

$$
j^{3}(E)=(\gamma / 2)\left(x^{2} y+x y^{2}\right), \quad \gamma=16 / 27 .
$$

That this is an elliptic umbilic is easily seen by setting $s=x+y, t=x-y$ giving us

$$
j^{3}(E)=(\gamma / 8)\left(s^{3}-s t^{2}\right)=(2 / 27)\left(s^{3}-s t^{2}\right)
$$

which appears on Thom's list with $\operatorname{det}[E]=3$.

Now consider the unfolding. Since $\operatorname{det}[E]=3$ we have $m^{4} \subset\langle m \mathcal{J}(E)\rangle$ where $\mathcal{J}(E)$ is the Jacobi ideal of $E$. Therefore $\mathcal{J}(E)$ is generated by the 3 -jets of the functions $E_{i}=\partial E / \partial u_{i}$. In $(x, y)$ coordinates this gives

$$
\mathcal{J}(E)=\left\langle 2 x y+y^{2}, x^{2}+2 x y\right\rangle_{\mathcal{E}}
$$


From this it follows that $m^{3} \subset\langle m \mathcal{J}(E)\rangle$. The quotient space $[m / \mathcal{J}(E)]$ is generated by the polynomials $\left\langle x, y, x^{2}+y^{2}\right\rangle$. Our unfolding directions are

$$
E_{r_{i}}\left(u_{1}, u_{2}, 1,1,1\right)-E_{r_{i}}(1,1,1,1,1), \quad i=1,2,3 .
$$

By the comments above we need only look at the 2-jet of these functions. Upon rewriting these functions using the $x-y$ coordinates we obtain

$$
\begin{aligned}
& T_{1}(x, y)=(-4 / 3) x+(8 / 9) x^{2}+\cdots \\
& T_{2}(x, y)=(-4 / 3) y+(8 / 9) y^{2}+\cdots \\
& T_{3}(x, y)=(4 / 3)(x+y)+(8 / 9)(x+y)^{2}+\cdots
\end{aligned}
$$

It remains to check that $m=\mathcal{J}(E) \oplus V(E)$ where $V(E)$ is the linear space generated by $T_{1}, T_{2}, T_{3}$ and $\mathcal{J}(E)$ is the ideal generated by $e_{1}, e_{2}$ as listed in (5.5). Since $m^{3} \subset\langle m \mathcal{J}(E)\rangle$ one needs only look at polynomials of degree at most two in $m$. This is a five dimensional space spanned by $\left\langle e_{1}, e_{2}, T_{1}, T_{2}, T_{3}\right\rangle$. Our unfolding is universal.

Note. From the discussion of Section 2 we know that a three small cap configuration $(s, s, s)$ is a stable local minimizer of energy while the three large cap configuration $(l, l, l)$ is unstable. At the triple hemisphere point $(h, h, h)$ the leading term in the energy function is the cubic (5.2) which shows that $(h, h, h)$ is not a local minimizer of energy. The configuration must travel to a new stable equilibrium.

We have identified $(1,1,1)$ on the bifurcation set $B_{E}$ corresponding to the $(h, h, h)$ configuration with the elliptic umbilic catastrophe. Three cusp lines pass through this point tangentially. One such cusp line lies in the plane $r_{1}=r_{2}$ and consists of those points $r_{1}=r_{2} \geq r_{3}$ supporting equilibrium configurations $(h, h, s)$ or $(h, h, l)$. At these points the second derivative will have rank one and the energy function will have determinacy greater than three. [Figure 4.]

Lemma 5.1. The cusp line for $E$ lying in the plane $r_{1}=r_{2}$ consists of those points $\left(r_{1}, r_{2}, r_{3}\right)$ where $r_{1}=r_{2} \geq r_{3} \geq 0$ supporting an equilibrium configuration of the form $(h, h, s)$ or $(h, h, l)$. Let branch $[A]$ correspond to those points supporting $(h, h, s)$. This branch connects $(1,1,1)$ to a point $\left(r_{A}, r_{A}, 0\right)$ where $r_{A}=(3 / 2)^{1 / 3} \cong 1.1447$. At this limit point the small cap has disappeared and $(h, h)$ consists of two hemispheres with $u_{1}+u_{2}=3$ or $V=2 \pi$. Branch $[B]$ are those points supporting $(h, h, l)$. It connects $(1,1,1)$ to a point $\left(r_{B}, r_{B}, 0\right)$ where the equilibrium configuration is two hemispheres plus an entire sphere of total volume $2 \pi$. This implies that $r_{B}=(3 / 4)^{1 / 3} \cong$ .9086. [Figure 5.]

Proof. A straightforward calculation. 
Theorem 5.2. Let $\left(r_{1}, r_{2}, r_{3}\right)$ be a point on the cusp curve $[A]$ with $r_{1}=r_{2}$ and $r_{3}=\lambda r_{1}, 0<\lambda<1$. Fix this point and consider the two families of equilibria with varying volume. One family is the symmetric family $\mathcal{C}_{1}$ : $(s, s, s) \rightarrow(h, h, s) \rightarrow(l, l, s)$ and the other is the bifurcating family $\mathcal{C}_{2}:$ $(s, l, s) \rightarrow(h, h, s) \rightarrow(l, s, s)$. As in Section 2 we parametrize each family by $t$ where $(0, t)$ locates the center of the first bubble with radius $a^{2}=r_{1}^{2}+t^{2}$. Let $V(t)$ be the total volume function for each family. For the symmetric family one finds that $V(t)$ is increasing with $\dot{V}(t)>0$ for all $t$. On the family $\mathcal{C}_{2}$ the volume function has the following properties.

(a) $V(t)$ is an even function of $t$ with limit $V(t)$ being $+\infty$ at each end.

(b) There is a value $\lambda^{*} \cong .98517$ such that:

(i) For $0<\lambda<\lambda^{*}$ the volume function has just one critical point at $t=0$ with $\dot{V}(0)=0$ and $\ddot{V}(0)>0$. For $t>0$ we have $\dot{V}(t)>0$ and for $t<0$ we have $\dot{V}(t)<0$.

(ii) For $\lambda^{*}<\lambda<1$ the volume function has three critical points. For $t=0$ we have $\dot{V}(0)=0$ and $\ddot{V}(0)<0$. The other critical points are at a value $t_{1}>0$ and $t_{2}=-t_{1}$. For $0<t<t_{1} \dot{V}(t)<0$ while for $t>t_{1}$ we have $\dot{V}(t)>0$.

(iii) $V(t)$ has a single critical point at $t=0$ when $\lambda=\lambda^{*}$. The second and third derivatives also vanish at $t=0$ but $V^{(4)}(0)$ is positive. Finally, $\dot{V}(t)>0$ for $t>0$ so that $V(t)$ has a minimum at $t=0$.

In other words if $0<\lambda<\lambda^{*}$ as one increases volume along the symmetric family $\mathcal{C}_{1}$ there will occur a forward bifurcation into stable non-symmetric equilibria while for $\lambda^{*}<\lambda<1$ there is a reverse bifurcation into unstable non-symmetric equilibria. In this case the state $(h, h, s)$ will fail to be a local minimizer. These two possibilities correspond to a cusp and its dual. Finally, as we shall see in Theorem 5.3, when $\lambda=\lambda^{*}$ there will occur a catastrophe of determinancy six (the Butterfly) at the $(h, h, s)$ point [Figure 5 and 6$]$.

Proof. Consider the symmetric family $\mathcal{C}_{1}$, with total volume $V=V_{1}+V_{2}+V_{3}$. From Theorem 2.1 we have that $d\left(V_{1}+V_{3}\right) / d t$ is positive. Since $V_{2}(t)$ is also increasing we see that $d V / d t$ is positive.

Now consider the non-symmetric family $\mathcal{C}_{2}$. The volume at any equilibrium is an entire sphere plus a small cap. From Section 2 one finds

$$
\begin{aligned}
V & =(\pi / 3)\left[6 a^{3}-3 a^{2} h+h^{3}\right] \\
a^{2} & =r_{1}^{2}+t^{2}, \quad h^{2}=a^{2}-r_{3}^{2}=\left(r_{1}^{2}-r_{3}^{2}\right)+t^{2} .
\end{aligned}
$$

If we set $r_{3}=\lambda r_{1}$ and $t=\tau r_{1}$ we find

$$
\dot{V}(t)=\pi \tau r_{1}^{2}\left[6 \sqrt{1+\tau^{2}}-\frac{\left(2-\lambda^{2}+2 \tau^{2}\right)}{\sqrt{\left(1-\lambda^{2}\right)+\tau^{2}}}\right]
$$




$$
\begin{aligned}
& =\pi \tau r_{1}^{2} \varphi(\lambda, \tau) \\
\ddot{V}(0) & =\pi r_{1}\left[6-\frac{2-\lambda}{\sqrt{1-\lambda^{2}}}\right]=\pi r_{1} \varphi(\lambda, 0) .
\end{aligned}
$$

From the second equation one concludes that there is exactly one value $\lambda^{*}$, $0<\lambda^{*}<1$, a root of $\lambda^{4}+32 \lambda^{2}-32=0$ such that $\ddot{V}(0)$ is positive for $0<\lambda<\lambda^{*}$ and is negative for $\lambda^{*}<\lambda<1$.

Consider the function $\varphi(\lambda, \tau)$ on the strip $0<\tau<\infty$ and $0 \leq \lambda \leq 1$. A close analysis reveals that $\varphi_{\tau}$ is positive on the strip. It follows that the zero set of $\varphi(\lambda, \tau)$ is given by a function $\tau=\tau(\lambda)$ defined for $\lambda^{*} \leq \lambda \leq 1$ connecting $\left(\lambda^{*}, 0\right)$ to $\left(1, \tau^{*}\right)$ for some $\tau^{*}>0$. It follows that for $0<\lambda<\lambda^{*}$, $\dot{V}(t)$ is positive when $t>0$ while for $\lambda^{*}<\lambda \leq 1 \dot{V}(t)$ is initially negative until we cross the zero set of $\varphi(\lambda, \tau)$ after which it is positive.

At $\lambda=\lambda^{*}$ we have $\ddot{V}(0)=0$ and moreover $\dddot{V}(0)=0$ as $V(t)$ is an even function of $t$. We claim that $V^{(4)}(0)$ is positive. Since $V(t)$ is an even function of $t$ we can express $V(t)=G\left(t^{2}\right)$ for any fixed $\lambda$. By the chain rule finds that $\ddot{V}(0)=2 G^{\prime}(0)$ and then $V^{(4)}(0)=12 G^{\prime \prime}(0)$. Again setting $r_{3}=\lambda r_{1}$ one obtains

$$
\begin{aligned}
G^{\prime}(0) & =\left(\pi r_{1} / 2\right)\left[6-\frac{2-\lambda^{2}}{\sqrt{1-\lambda^{2}}}\right]=\frac{\pi r_{1}}{2} \varphi(\lambda, 0) \\
G^{\prime \prime}(0) & =\frac{\pi}{4 r_{1}}\left[6+\frac{3 \lambda^{2}-2}{\left(1-\lambda^{2}\right)^{\frac{3}{2}}}\right] .
\end{aligned}
$$

When $\lambda=\lambda^{*}$ and $G^{\prime}(0)=0$ one sees that $G^{\prime \prime}(0)$ is positive whence $V^{(4)}(0)$ is also positive.

Now we shall compute more explicitly the energy function $E$ along the cusp line $[A]$. We introduce an explicit change of coordinates so that the 4 -jet of the energy function splits into a non-degenerate part and a totally degenerate part, making obvious the nature of the catastrophe. We do this with a view to examing the unfolding introduced by our three parameters.

Theorem 5.3. For each point $\left(r_{1}, r_{2}, r_{3}\right)$ on the cusp curve $[A]$ with equilibrium configurations of the form $(h, h, s)$ there exists a smooth change of coordinates $\left(u_{1}, u_{2}\right) \rightarrow\left(w_{1}, w_{2}\right)$ which is a local diffeomorphism and is a polynomical function such that the 4-jet of the energy $E$ (up to a constant) on the cusp line $[A]$ takes the form

$$
\begin{aligned}
j^{4}(E) & =w_{1}^{2}+D w_{2}^{4}, \quad D=C-1 \\
\text { where } C & =(9 / 2)\left(r_{1} / r_{3}\right)^{4} F^{\prime \prime}\left(\widehat{u}_{3} / r_{3}^{3}\right) .
\end{aligned}
$$

If $D \neq 0$ then $\operatorname{det}[E]=4$ and we have, after splitting off the $w_{1}$ coordinate, the standard cusp catastrophe. The direction of the bifurcation is determined by the sign of $D$. At exactly one point on the curve $[A] D$ will vanish. At 
this point (corresponding to $\left.\lambda=\lambda^{*}\right) \operatorname{det}[E]=6$ and one can introduce coordinates so that

$$
j^{6}(E)=w_{1}^{2}+F w_{2}^{6} \quad \text { where } F>0 .
$$

At this point the energy has, after splitting off the first coordinate, a butterfly catastrophe requiring four parameters for a universal unfolding. The expressions $j^{4}(E)$ and $j^{6}(E)$ are evaluated at $\widehat{u}_{1}=r_{1}^{3}, \widehat{u}_{2}=r_{2}^{3}$ and $\widehat{u}_{1}+\widehat{u}_{2}+\widehat{u}_{3}=3$ where $\left(r_{1}, r_{2}, r_{3}\right)$ with $r_{1}=r_{2}>r_{3}$ lies on the cusp line $[A]$.

Proof. Let $\left(r_{1}, r_{1}, r_{3}\right)$ lie on the cusp curve $[A]$ supporting a configuration $(h, h, s)$. Let $\left(\widehat{u}_{1}, \widehat{u}_{2}, r_{1}, r_{1}, r_{3}\right)$ be the corresponding point on the catastrophe set $C_{E}$ so that $\widehat{u}_{1}=\widehat{u}_{2}=r_{1}^{3}, \widehat{u}_{3} / r_{3}^{3}<1$ and $\widehat{u}_{1}+\widehat{u}_{2}+\widehat{u}_{3}=3$. The energy $E$ is given by (5.1). We want to expand $E$ about $\left(\widehat{u}_{1}, \widehat{u}_{2}\right)$ in powers of $\left(u_{1}-\widehat{u}_{1}, u_{2}-\widehat{u}_{2}\right)$. If we set $x=u_{1}-\widehat{u}_{1}$ and $y=u_{2}-\widehat{u}_{2}$ then the $n$-th order term in the power series expansion of $E$ is

$$
\begin{aligned}
& E_{n}=\frac{1}{n !}\left[A_{n}\left(x^{n}+y^{n}\right)+(-1)^{n} B_{n}(x+y)^{n}\right] \\
& A_{n}=\frac{1}{r_{1}^{3 n-2}} F^{(n)}(1), \quad B_{n}=\frac{1}{r_{3}^{3 n-2}} F^{(n)}\left(\widehat{u}_{3} / r_{3}^{3}\right) .
\end{aligned}
$$

Noting that $A_{2}=0$ as $F^{\prime \prime}(1)=0$ we find the 4 -jet of $E$ (up to a constant) to be

$$
\begin{aligned}
\frac{B_{2}}{2}(x+y)^{2}+ & \frac{1}{6}\left[A_{3}\left(x^{3}+y^{3}\right)-B_{3}(x+y)^{3}\right] \\
& +\frac{1}{24}\left[A_{4}\left(x^{4}+y^{4}\right)+B_{4}(x+y)^{4}\right] .
\end{aligned}
$$

On the curve $[A] B_{2}$ is positive (while on the other curve $[B]$ the factor $B_{2}$ is negative). We simplify (5.11) by first making a linear change of coordinates $s=x+y, \sigma t=x-y$ getting

$$
\begin{aligned}
& j^{4}(E)=\frac{a}{2}\left\{s^{2}+\left(-2 s t^{2}+2 \alpha s^{4}\right)+\left(A s^{4}+B s^{2} t^{2}+C t^{4}\right)\right\} . \\
& \text { Here } a=B_{2}, \sigma^{2}=-8 a / A_{3}, A_{3}=\frac{1}{r_{1}^{7}} F^{\prime \prime \prime}(1)=-\gamma / r_{1}^{7}<0 \\
& 2 \alpha=\frac{A_{3}-4 B_{3}}{12 a}, A=\frac{A_{4}+8 B_{4}}{96 a}, B=\frac{\sigma^{2} A_{4}}{16 a}, C=\frac{\sigma^{4} A_{4}}{96 a} .
\end{aligned}
$$

On the cusp curve $[A] a=B_{2}$ is positive and so $D_{u}^{2} E$ has rank one. We complete the splitting at the 4 -jet level by using a device found in Poston and Stewart [10]. The cubic term $Q_{3}$ in (5.12) has a factor $s$, so we write $Q_{3}=2 s R_{2}$ where $R_{2}=\alpha s^{2}-t^{2}$. Define the transformation $\left(v_{1}, v_{2}\right)=$ $\Phi_{1}(s, t)$ by

$$
v_{1}=s+R_{2}=s+\left(\alpha s^{2}-t^{2}\right), \quad v_{2}=t .
$$


Note that $\Phi_{1}$ is a local diffeomorphism with $D \Phi_{1}(0)=I d$. To compute the inverse of the map $\Phi_{1}$ we note that $t=v_{2}$ and $\alpha s+s=\left(v_{1}+v_{2}^{2}\right)$. With the aid of the quadratic formula and the binomial series we find

$$
\begin{aligned}
s= & \left(v_{1}+v_{2}^{2}\right)-\alpha\left(v_{1}+v_{2}^{2}\right)^{2}+2 \alpha^{2}\left(v_{1}+v_{2}^{2}\right)^{3} \\
& -5 \alpha^{3}\left(v_{1}+v_{2}^{2}\right)^{4}+\cdots \\
t= & v_{2} .
\end{aligned}
$$

We can now rewrite (5.12) as

$$
j^{4}(E)=\frac{a}{2}\left\{v_{1}^{2}+\left[\left(A-\alpha^{2}\right) v_{1}^{4}+(B+2 \alpha) v_{1}^{2} v_{2}^{2}\right]+(C-1) v_{2}^{4}\right\} .
$$

The expression in brackets has a $v_{1}$ factor so we write it as $2 v_{1} R_{3}$. Now define $\left(w_{1}, w_{2}\right)=\Phi_{2}\left(v_{1}, v_{2}\right)$ by

$$
\begin{aligned}
& w_{1}=v_{1}+R_{3}=v_{1}+\frac{1}{2}\left[\left(A-\alpha^{2}\right) v_{1}^{3}+(B+2 \alpha) v_{1} v_{2}^{2}\right] \\
& w_{2}=v_{2} .
\end{aligned}
$$

Again $\Phi_{2}$ is a local diffeomorphism with $D \Phi_{2}(0)=I d$. We end up with

$$
j^{4}(E)=\frac{a}{2}\left\{w_{1}^{2}+(C-1) w_{2}^{4}\right\} .
$$

Using the fact that $F^{\prime \prime \prime}(1)=-\gamma=-16 / 27$ and $F^{(4)}(1)=\delta=64 / 27$ and the formulas in (5.12) we obtain

$$
C=(9 / 2)\left(r_{1} / r_{3}\right)^{4} F^{\prime \prime}\left(\widehat{u}_{3} / r_{3}^{3}\right) .
$$

As $\lambda$ increases from 0 to 1 the ratio $\widehat{u}_{3} / r_{3}^{3}$ does the same as the small cap of $(h, h, s)$ expands into a hemisphere. Since $F^{\prime \prime \prime}(t)$ is negative it follows that $F^{\prime \prime}(t)$ is decreasing on the interval $(0,1)$. We conclude that $C$ is decreasing on $0<\lambda<1$ from $+\infty$ to 0 . It equals one for a single value $\lambda=\lambda_{1}$. We claim that $C=1$ precisely when $\lambda=\lambda^{*}$ where $\lambda^{*}$ is that value determined in Theorem 5.2. Thus, for $0<\lambda<\lambda^{*}$ the quantity $C-1$ is positive and the bifurcation is forward while for $\lambda^{*}<\lambda<1$ the quantity $C-1$ is negative and the direction of bifurcation is reversed. Finally, we shall show that at the point $P^{*}=\left(r_{1}^{*}, r_{1}^{*}, r_{3}^{*}\right)$ where $C=1$ the energy function $E$ has determinancy six and in suitable coordinates takes the form (5.9).

We compute the 6 -jet of $E$ at the point on the cusp curve $[A]$ where $C=1$, proceeding in the same manner as in the calculation of the 4-jet, by making further $C^{\infty}$ changes of coordinates until the desired form is reached. Using the same notation as in (5.10-5.12) we find the 6-jet of $E$ (up to a constant) to be

$$
\begin{aligned}
\frac{B_{2}}{2}(x+y)^{2} & +\frac{1}{6}\left[A_{3}\left(x^{3}+y^{3}\right)-B_{3}(x+y)^{3}\right] \\
& +\frac{1}{24}\left[A_{4}\left(x^{4}+y^{4}\right)+B_{4}(x+y)^{4}\right]
\end{aligned}
$$




$$
\begin{aligned}
& +\frac{1}{120}\left[A_{5}\left(x^{5}+y^{5}\right)-B_{5}(x+y)^{5}\right] \\
& +\frac{1}{720}\left[A_{6}\left(x^{6}+y^{6}\right)+B_{6}(x+y)^{6}\right] .
\end{aligned}
$$

Once again we rewrite this expression by setting $s=x+y, \sigma t=x+y$ to obtain

$$
\begin{aligned}
j^{6}(E)= & \frac{a}{2}\left\{s^{2}+\left(-2 s t^{2}+2 \alpha s^{3}\right)\right. \\
& +\left(A s^{4}+B s^{2} t^{2}+C t^{4}\right)+\left(D s^{5}+E s^{3} t^{2}+F s t^{4}\right) \\
& \left.+\left(G s^{6}+H s^{4} t^{2}+I s^{2} t^{4}+J t^{6}\right)\right\} .
\end{aligned}
$$

The constants $a, \sigma^{2}, \alpha, A, B, C$ are displayed in (5.12). The remaining constants are calculated in a similar fashion. In particular we need to observe

$$
J=\left(\frac{2}{a}\right)\left(\frac{\sigma^{6} A_{6}}{(720)(64)}\right)=\frac{\sigma^{6} A_{6}}{23040 a}
$$

where we have made use of the identity

$$
x^{6}+y^{6}=\frac{1}{64}\left(s^{6}+15 \sigma^{2} s^{4} t^{2}+15 \sigma^{4} s^{2} t^{4}+\sigma^{6} t^{6}\right) .
$$

Now make the transformation $\left(v_{1}, v_{2}\right)=\Phi_{1}(s, t)$ given by (5.13) to rewrite $j^{6}(E)$ as

$$
\begin{aligned}
j^{6}(E)= & \frac{a}{2}\left\{v_{1}^{2}+\left[\left(A-\alpha^{2}\right) v_{1}^{4}+(B+2 \alpha) v_{1}^{2} v_{2}^{2}+(C-1) v_{2}^{4}\right]\right. \\
& \left.+v_{1} P_{4}\left(v_{1}, v_{2}\right)+v_{1} P_{5}\left(v_{1}, v_{2}\right)+(2 \alpha+B+J) v_{2}^{6}\right\} .
\end{aligned}
$$

Here $P_{4}\left(v_{1}, v_{2}\right)$ and $P_{5}\left(v_{1}, v_{2}\right)$ are homogeneous polynomials of degree four and five, respectively. We note that there is a factor $v_{1}$ in front of these terms. If $C-1 \neq 0$ we need only consider the 4 -jet and arrive at the expression (5.17). We continue with the case $C-1=0$. The successor transformation $\left(w_{1}, w_{2}\right)=\Phi_{2}\left(v_{1}, v_{2}\right)$ is given by (5.16). Rewrite the expression (5.22) in the new coordinates. Recall that $v_{1}^{2}+2 v_{1} R_{3}=w_{1}^{3}-R_{3}^{2}$ and that the inverse transformation has the form

$$
\begin{aligned}
& v_{1}=w_{1}-R_{3}\left(w_{1}, w_{2}\right)+[\text { higher order terms] } \\
& v_{2}=w_{2}
\end{aligned}
$$

leading to

$$
\begin{aligned}
j^{6}(E)= & \frac{a}{2}\left\{w_{1}^{2}-\frac{1}{4} w_{1}^{2}\left[\left(A-\alpha^{2}\right) w_{1}^{2}+(B+2 \alpha) w_{2}^{2}\right]^{2}\right. \\
& \left.+w_{1} P_{4}\left(w_{1}, w_{2}\right)+w_{1} P_{5}\left(w_{1}, w_{2}\right)+(2 \alpha+B+J) w_{2}^{6}\right\} \\
= & \frac{a}{2}\left\{w_{1}^{2}+2 w_{1} Q_{4}\left(w_{1}, w_{2}\right)+(2 a+B+J) w_{2}^{6}\right\}
\end{aligned}
$$


where $Q_{4}\left(w_{1}, w_{2}\right)$ is a polynomial each of whose terms are of degreee at least four. We make a final change of coordinates

$$
\begin{aligned}
& w_{1}^{\prime}=w_{1}+Q_{4}\left(w_{1}, w_{2}\right) \\
& w_{2}^{\prime}=w_{2}
\end{aligned}
$$

keeping in mind that $Q_{4}\left(w_{1}, w_{2}\right)$ is of degree at least four and that $w_{1}^{2}+$ $2 w_{1} Q_{4}\left(w_{1}, w_{2}\right)=w^{\prime 2}-Q_{4}^{2}\left(w_{1}, w_{2}\right)$ we arrive at

$$
j^{6}(E)=\frac{a}{2}\left\{w_{2}^{2}+(2 \alpha+B+J) w_{2}^{6}\right\}
$$

where (for convenience) we have deleted the primes in the formula and we have suppressed the additive constant.

We need to show that $2 \alpha+B+J$ is positive. In fact, we will find that each term in this expression is positive.

At this point we need to verify that the condition $C=1(5.18)$ occurs precisely when $\lambda=r_{3} / r_{1}$ satisfies the polynomial identity $\lambda^{4}+32 \lambda^{2}-32=0$, $0<\lambda<1$ as given in Theorem 5.2. From the equilibrium condition of $(h, h, s)$ along the cusp curve $[A]$ one has

$$
F^{\prime}\left(u_{3} / r_{3}^{3}\right)=\left(r_{3} / r_{1}\right) F^{\prime}(1)=4 \lambda / 3 .
$$

Now assume $\lambda=\lambda^{*}$ is a root of the polynomial equation $\lambda^{4}+32 \lambda^{2}-32=0$, $0<\lambda<1\left(\lambda^{*} \cong .98517\right)$. We want to show

$$
F^{\prime \prime}\left(\widehat{u}_{3} / r_{3}^{3}\right)=\frac{2}{9}\left(r_{3} / r_{1}\right)^{4}=\frac{2 \lambda^{4}}{9} .
$$

Recall that $F(u)=\frac{1}{\pi} a\left(\frac{2 \pi u}{3}\right)$ where $a(v)$ is expressed as a function of $t$ in (2.2). It follows that $F^{\prime}(u)=2 a^{\prime}(v) / 3=4 / 3 \sqrt{1+t^{2}}$ using (2.3), leading to the relation $\lambda^{2}\left(1+t^{2}\right)=1$ where $t$ is negative $\left(t^{*} \cong-.17416\right)$. We have $F^{\prime \prime}(u)=\frac{4 \pi}{9} a^{\prime \prime}\left(\frac{2 \pi u}{3}\right)$. Now use the expression for $a^{\prime \prime}(v)$ given in (2.3) to obtain

$$
F^{\prime \prime}\left(\widehat{u}_{3} / r_{3}^{3}\right)=-\frac{8}{9}\left[t /\left(1+t^{2}\right)^{3 / 2}\right]\left(\sqrt{1+t^{2}}-t\right)^{2} .
$$

Since $\lambda^{2}\left(1+t^{2}\right)=1$ with $t<0$ we have $t=-\sqrt{1-\lambda^{2}} / \lambda$ and find

$$
F^{\prime \prime}\left(\widehat{u}_{3} / r_{3}^{3}\right)=\frac{8}{9}\left(1-\lambda^{2}\right)\left[\frac{2-\lambda^{2}}{\sqrt{1-\lambda^{2}}}+2\right] \text {. }
$$

By (5.7b) we observe that $\left(2-\lambda^{2}\right) / \sqrt{1-\lambda^{2}}=6$ and so

$$
F^{\prime \prime}\left(\widehat{u}_{3} / r_{3}^{3}\right)=\frac{64}{9}\left(1-\lambda^{2}\right)=\frac{2 \lambda^{4}}{9}
$$

showing that $C=1$ when $\lambda=\lambda^{*}$.

We now compute $2 \alpha=\left(A_{3}-4 B_{3}\right) / 12 a$ where $a=B_{2}$ is positive and $A_{3}, B_{3}$ are given in (5.10). Using the fact that $F^{\prime \prime}(1)=-16 / 27$ and $r_{3} / r_{1}=$ 
$\lambda$ we obtain

$$
A_{3}-4 B_{3}=\frac{1}{r_{1}^{7}}\left[F^{\prime \prime \prime}(1)-\frac{4}{\lambda^{7}} F^{\prime \prime \prime}\left(u_{3} / r_{3}^{3}\right)\right]=\frac{-4}{27 r_{3}^{3}}\left[4 \lambda^{7}+27 F^{\prime \prime \prime}\left(u_{3} / r_{3}^{3}\right)\right] .
$$

However $F^{\prime \prime \prime}(u)=\frac{8 \pi^{2}}{27} a^{\prime \prime \prime}\left(\frac{2 \pi u}{3}\right)$ and upon using (2.3) we find

$$
A_{3}-4 B_{3}=\frac{-16}{27 r_{1}^{7}}\left\{1-4\left(1+t^{2}\right)\left(\sqrt{1+t^{2}}-t\right)^{4}\left(1-2 t^{2}-2 t \sqrt{1-t^{2}}\right)\right\} \text {. }
$$

If we evaluate this quality for $t^{*} \cong-.17416$ we find

$$
A_{3}-4 B_{3} \cong 5.722 / r_{1}^{7} \text {. }
$$

Now $a=F^{\prime \prime}\left(u_{3} / r_{3}^{3}\right) / r_{3}^{4}=2 / 9 r_{1}^{4}$ when $C=1$ giving us

$$
2 \alpha=\frac{A_{3}-4 B_{3}}{12 a} \cong 2.146 / r_{1}^{3} \text {. }
$$

We next calculate $B=\sigma^{2} A_{4} / 16 a$. Here $A_{4}=F^{(4)}(1) / r_{1}^{10}=64 / 27 r_{1}^{10}$, $\sigma^{2}=-8 a / A_{3}$ with $a=B_{2}=2 / 9 r_{4}^{1}$. Clearly $B$ is positive and we find

$$
B=2 / r_{1}^{3} \text {. }
$$

Finally we calculate $J=\sigma^{6} A_{6} / 20340 a$. Substituting $\sigma^{2}=-8 a / A_{3}$ with $a=2 / 9 r_{1}^{4}$ when $C=1$ leads to

$$
J=\frac{27}{5120} \frac{F^{(6)}(1)}{r_{1}^{3}} .
$$

The calculation of $F^{(6)}(1)$ is based on the identity $F^{(6)}(u)=\frac{1}{\pi}\left(\frac{2 \pi}{3}\right)^{6} a^{(6)}\left(\frac{2 \pi u}{3}\right)$. We use the formula for $a^{(4)}(v)$ given as a function of $t$ in (2.3). Call $a^{(4)}(v) \equiv$ $\theta(t)$ and use the expression for $\dot{v}(t)$ as displayed in (2.2). We have

$$
a^{(6)}(2 \pi / 3)=\frac{\dot{v}(0) \ddot{\theta}(0)-\dot{\theta}(0) \ddot{v}(0)}{\dot{v}(0)^{3}} .
$$

We see that $\dot{v}(0)=\pi, \ddot{v}(0)=2 \pi$ and also

$$
\theta(t)=\frac{2}{\pi^{3}}\left\{6-35 t+67 t^{2}+\cdots\right\} .
$$

Our calculations give $F^{(6)}(1)=8576 / 243$ leading to

$$
J=\frac{67}{360} \cdot \frac{1}{r_{1}^{3}} .
$$

The quantity $2 \alpha+B+J$ is positive and the 6-jet of $E$ has the form (5.9).

At the point $P^{*}$ the state $(h, h, s)$ determines a butterfly with $\operatorname{det}[E]=6$. We note that this $(h, h, s)$ state is a local (and global) minimizer of energy. From our table we see that $\operatorname{cod}[E]=4$ and so any universal unfolding requires four parameters. Three parameters $\left(r_{1}, r_{2}, r_{3}\right)$ are at hand. One more is needed. As discussed in the introduction any attempt to introduce 
a fourth unfolding parameter by perturbing the boundary curves away from circles fails.

\section{The Butterfly Unfolding.}

We now shall introduce a fourth unfolding parameter for the energy at the point $P^{*}$ where there is a butterfly catastrophe for the configuration $(h, h, s)$. Three unfolding parameters are provided by $\left(r_{1}, r_{2}, r_{3}\right)$. A fourth unfolding parameter will first be developed by adding a gravitational potential to the exposed fluid mass. This will give new equilibrium configurations whose surfaces will have prescribed mean curvature and which we represent as a normal graph over the upper hemisphere, [Theorem 6.2]. Following this we consider the effect of varying the surface tensions of the liquid-air interfaces and show that this also yields a universal unfolding at the butterfly point, [Theorem 6.3].

Definition 6.1. Let $\rho(\varphi, \theta)=1+f(\varphi, \theta)$ describe a surface given by a radial graph over the hemisphere which in spherical coordinates is given by $\rho=1,0 \leq \varphi \leq \pi / 2,0 \leq \theta \leq 2 \pi$. For such a normal graph we have

$$
\begin{aligned}
A & =\int_{0}^{2 \pi} \int_{0}^{\pi / 2} \rho \sqrt{\rho_{\theta}^{2}+\left(\rho^{2}+\rho_{\varphi}^{2}\right) \sin ^{2} \varphi} d \varphi d \theta, \\
V & =\frac{1}{3} \int_{0}^{2 \pi} \int_{0}^{\pi / 2} \rho^{3} \sin \varphi d \varphi d \theta, \\
P & =\frac{1}{4} \int_{0}^{2 \pi} \int_{0}^{\pi / 2} \rho^{4} \sin \varphi \cos \varphi d \varphi d \theta .
\end{aligned}
$$

Here $A$ is the surface area, $V$ is the volume enclosed by the surface along with the planar region spanning its boundary and $P$ is a gravitational potential energy with the force acting vertically with intensity $\epsilon$. We assume that $\rho=1+f$ where $f \in C^{2, \alpha}\left(\Sigma_{0}\right)$. We can identify $\Sigma_{0}$ with the unit disk and shall usually represent points on $\Sigma_{0}$ using spherical polar coordinates.

$$
\Sigma_{0}=\left\{x \in R^{3} \mid x^{2}+y^{2}+z^{2}=1, \quad z \geq 0\right\} .
$$

For any $\epsilon$ and $f \in C_{0}^{2, \alpha}\left(\Sigma_{0}\right)$ we define the perturbed energy by

$$
E \equiv A(\rho)+\epsilon P(\rho), \quad \rho=1+f .
$$

For general $\epsilon$ we look for local minimizers of the energy $E$ subject to the volume constraint $V(f)=v$. By the method of Lagrange multipliers there is a constant $k$ such that for any $h \in C_{0}^{2, \alpha}\left(\Sigma_{0}\right)$ the first variation of the energy will vanish.

$$
\partial[A+\epsilon P+k V](f)(h)=0
$$


The following theorem establishes the existence of solutions to this problem for $(\epsilon, v)$ close to $(0,2 \pi / 3)$. The Euler-Lagrange equations in spherical coordinates take the form

$$
\begin{aligned}
M(\rho)+ & \epsilon(\rho \cos \varphi)+k=0, \quad \rho=1+f \\
M(\rho)= & \frac{1}{\rho^{2} \sin \varphi}\left\{-\frac{\partial}{\partial \varphi}\left(\frac{\rho \rho_{\varphi} \sin ^{2} \varphi}{W}\right)-\frac{\partial}{\partial \theta}\left(\frac{\rho \rho_{\theta}}{W}\right)\right. \\
& \left.+\frac{\rho_{\theta}^{2}+\left(2 \rho^{2}+\rho_{\varphi}^{2}\right) \sin ^{2} \varphi}{W}\right\}
\end{aligned}
$$

where $W=\sqrt{\rho_{\theta}^{2}+\left(\rho^{2}+\rho_{\varphi}^{2}\right) \sin ^{2} \varphi} \cdot M(\rho)$ is the mean curvature operator with $M(\rho)=2 H$ where $H$ is the mean curvature of the surface. The function $\rho(\varphi, \theta)$ is an extremal when the corresponding surface $\Sigma_{\rho}$ has the property that its mean curvature satisfies the relation $2 H+\epsilon z+k=0$ where $(\epsilon, k)$ are constants and $z$ is the vertical coordinate.

Theorem 6.1. Consider the function $\mathcal{F}: C_{0}^{2, \alpha}\left(\Sigma_{0}\right) \times R^{2} \rightarrow C^{\alpha}\left(\Sigma_{0}\right) \times R$ defined by

$$
\mathcal{F}(f, \epsilon, k)=\langle M(\rho)+\epsilon z+k, V(\rho)\rangle, \quad \rho=1+f .
$$

Note that $\mathcal{F}(0,0,-2)=(0,2 \pi / 3)$. For each choice of $(\epsilon, v)$ near $(0,2 \pi / 3)$ there exists a uniquely determined pair of smooth functions $g: U \rightarrow C_{0}^{2, \alpha}\left(\Sigma_{0}\right)$ and $k: U \rightarrow R$ where $U \subset R^{2}$ is a neighborhood of $(\epsilon, v)=(0,2 \pi / 3)$ such that the set of solutions to $\mathcal{F}(f, \epsilon, k)=(0, v)$ in a neghborhood of $(\epsilon, v)=(0,2 \pi / 3)$ when $g=0, k=-2$ is given by the pair $\langle g, k\rangle$. In other words, for a given volume $v$ near $2 \pi / 3$ and gravitational potential energy with intensity $\epsilon$ near zero there is a surface $\Sigma_{\rho}$ represented by a normal graph over the hemisphere whose radial function satisfies the Euler-Lagrange Equation (6.4), spans the unit circle and encloses the desired volume.

Proof. The proof is an application of the inverse function theorem. We shall compute the derivative of $\mathcal{F}$ in the direction $(f, k)$ evaluated at $(f, \epsilon, k)=$ $(0,0,-2)$ and show that the corresponding linear operator mapping $C_{0}^{2, \alpha}\left(\Sigma_{0}\right)$ $\times R$ into $C^{\alpha}\left(\Sigma_{0}\right) \times R$ is invertible. It is a Fredholm operator (by general elliptic theory) and we shall prove directly that it has a trivial kernel and is surjective. We compute

$$
\begin{aligned}
& D_{(f, k)} \mathcal{F}[f=0, \epsilon=0 k=-2](h, \dot{k}) \\
& =\left\langle-\frac{1}{\sin \varphi}\left[\frac{\partial}{\partial \varphi}\left(\sin \varphi h_{\varphi}\right)+\frac{\partial}{\partial \theta}\left(\frac{h_{\theta}}{\sin \varphi}\right)\right]-2 h+\dot{k},\right. \\
& \left.\int_{0}^{2 \pi} \int_{0}^{\pi / 2} h \sin \varphi d \varphi d \theta\right\rangle
\end{aligned}
$$




$$
=\left\langle-\Delta h-2 h+\dot{k}, \iint h d A\right\rangle, h \in C_{0}^{2, \alpha}\left(\Sigma_{0}\right), \dot{k} \in \mathbb{R} .
$$

Here $\Delta h$ is the surface Laplacian on the upper unit hemisphere $\Sigma_{0}$ and integration is taken over this surface. We claim that the kernel of $D \mathcal{F}$ is $(h, \dot{k})=(0,0)$. Suppose that $(h, \dot{k})$ is in the kernel with $\dot{k}=0$ so that $\Delta h+2 h=0$ on $\Sigma_{0}$ with $h=0$ on $\partial \Sigma_{0}$. This means that $h$ is a spherical harmonic vanishing on the equator. The only solution up to a scalar factor is $h_{0}(x)=z$. Since $h_{0}(x)$ is positive on $\Sigma_{0}$ we have $\iint h_{0} d A$ is positive violating the second condition. Now suppose $(h, \dot{k})$ is in the kernel with $\dot{k} \neq 0$ and $\iint h_{0} d A=0$. One obtains two identities involving $h_{0}, h$ and $\dot{k}$ by multiplying the respective PDE's satisfied by $h_{0}$ and $h$ by the other function and integrating by parts, obtaining

$$
\begin{aligned}
-D\left(h_{0}, h\right)+2 \iint h_{0} h d A & =0 \\
-D\left(h_{0}, h\right)+2 \iint h h_{0} d A-\dot{k} \iint h_{0} d A & =0
\end{aligned}
$$

where $D\left(h_{0}, h\right)$ is the Dirichlet inner product of $h_{0}$ and $h$. This is not possible unless $\dot{k}=0$. The kernel of $D \mathcal{F}$ is trivial. Now we show that the range of $D \mathcal{F}$ is all of $C^{\alpha}\left(\Sigma_{0}\right) \times R$. Select $w(x) \in C^{\alpha}\left(\Sigma_{0}\right)$ and $v \in R$. We are to show that there is a pair $(h, \dot{k})$ with $-\Delta h-2 h+\dot{k}=w(x)$ and $\iint h d A=v$ where $h \in C_{0}^{2, \alpha}\left(\Sigma_{0}\right)$. The PDE $\Delta h+2 h=\dot{k}-w(x) \equiv r(x)$ has a solution $h(x) \in C_{0}^{2, \alpha}\left(\Sigma_{0}\right)$ precisely when $r(x)$ is orthogonal to the eigenfunction $h_{0}(x)=z$. This means

$$
\iint h_{0}(\dot{k}-w(x)) d x=0
$$

Since $h_{0}(x)=z$ is positive we have determined a unique value $\dot{k}$. Now let $h_{1}(x)$ be a solution to $\Delta h_{1}+2 h_{1}=\dot{k}-w(x)$. Then so is $h_{\lambda}(x)=$ $h_{1}(x)+\lambda h_{0}(x)$ for any $\lambda$. But we are to have

$$
\iint h_{\lambda} d A=\iint h_{1} d A+\lambda \iint h_{0} d A=v .
$$

Since $h_{0}(x)$ is positive there is a unique value of $\lambda$ so that the pair $\left(h_{\lambda}, \dot{k}\right)$ solves our problem. The map $D \mathcal{F}$ is surjective and thus is invertible. Since $\mathcal{F}$ is a differentiable map we can apply the implicit function theorem to complete the proof.

Corollary. There is a smooth function $E(v, \epsilon)$ defined in a neighborhood of $(2 \pi / 3,0)$ by

$$
\begin{aligned}
E(v . \epsilon) & =A(v, \epsilon)+\epsilon P(v, \epsilon) \\
\text { where } A(v, \epsilon) & =A[\rho(\varphi, \theta, \epsilon, v)]
\end{aligned}
$$




$$
P(v, \epsilon)=P[\rho(\varphi, \theta, \epsilon, v)]
$$

where $\rho=1+g$ with $g \in C_{0}^{2, \alpha}\left(\Omega_{0}\right)$ is determined from Theorem 6.1 with $V[\rho(\varphi, \theta, \epsilon, v)]=v$ and the integrals are given by (6.1).

Note that for fixed $\epsilon, g(\varphi, \theta, \epsilon, v)$ is an extremal for the volume constrained problem with energy $E(\rho, \epsilon)=A(\rho)+\epsilon P(\rho)$ where $\rho=1+g$. It follows that

$$
A_{\epsilon}(v, \epsilon)+\epsilon P_{\epsilon}(v, \epsilon)=0,
$$

as a result of which we find

$$
\frac{\partial}{\partial \epsilon}[A(v, \epsilon)+\epsilon P(v, \epsilon)]=P(v, \epsilon) .
$$

When $\epsilon=0$ the spherical cap provides the area minimizer for a given volume. This allowed us to reduce the configuration space for a multiple orifice problem from an infinite dimensional setting to one of finite dimensions by replacing any configuration enclosing a given volume and spanning a circle with the corresponding spherical cap of lower energy. For $\epsilon \neq 0$ the role of spherical caps are replaced by $\epsilon$-extremals of Theorem 6.1 which are sessile drops when $\epsilon$ is positive and pendant drops for negative $\epsilon$. When $\epsilon \neq 0$ these extrema are never global minimizers but they are stable local minimizers of the energy. Thus for $\epsilon \neq 0$ we also have a finite dimensional configuration space. For each opening the spherical cap is replaced by the corresponding sessile or pendant drop. These drops are rotationally symmetric and are strong local minimizers of energy. For a more complete discussion see [11].

Theorem 6.2. Let $P^{*}=\left(r_{1}^{*}, r_{1}^{*}, r_{3}^{*}\right)$ be that point on the cusp curve $[A]$ of the bifurcation set where the butterfly catastrophe occurs.

$A$ universal unfolding of the butterfly is given by

$$
E\left(u_{1}, u_{2}, r_{1}, r_{2}, \epsilon\right)=E\left(u_{1}, r_{1}, \epsilon\right)+E\left(u_{2}, r_{2},-\epsilon\right)+E\left(u_{3}, r_{3}, 0\right)
$$

where $u_{i}$ are the scaled volumes $u_{1}+u_{2}+u_{3}=3$ and we write

$$
E(u, r, \epsilon)=\frac{1}{\pi}[A(u, r, \epsilon)+\epsilon P(u, r, \epsilon)]
$$

where upon making use of scaling properties we have

$$
\begin{aligned}
& A(u, r, \epsilon)=r^{2} A\left(v, r^{2} \epsilon\right) \\
& P(u, r, \epsilon)=r^{4} P\left(u, r^{2} \epsilon\right), \quad v=(2 \pi / 3) r^{-3} u .
\end{aligned}
$$

We have divided by $\pi$ to be consistent with Definition 2.1. The apparatus is arranged so that if the first drop is disturbed by positive gravity then the second is disturbed in the opposite direction.

Proof. From the proof of Theorem 5.3 there is a change of coordinates $\left(u_{1}, u_{2}\right) \rightarrow(x, y) \rightarrow(s, t) \rightarrow\left(w_{1}, w_{2}\right)$ where $x=u_{1}-u_{1}^{*}, y=u_{2}-u_{2}^{*}$, $s=x+y, \sigma t=x-y$ and the entire map is a local diffeomorphism of polynomial type with $w_{2}=t$. Recall that the scaled volumes at $P^{*}$ are $\left(u_{1}^{*}, u_{2}^{*}, u_{3}^{*}\right)$ 
where $u_{1}^{*}=u_{2}^{*}=r_{1}^{* 3}$. At $P^{*}$ we have the butterfly with $j^{6}(E)=w_{1}^{2}+F w_{2}^{6}$ and $F>0$. The Jacobi ideal $\mathcal{J}(E)=\left\langle w_{1}, w_{2}^{5}\right\rangle_{\mathcal{E}}$, and so the space $m \bmod$ $\mathcal{J}(E)$ is spanned by $\left\langle w_{2}, w_{2}^{2}, w_{2}^{3}, w_{2}^{4}\right\rangle$ with $\operatorname{cod}[E]=4$. To verify universality of the unfolding we need to show that the four functions obtained by differentiating the energy $E$ (6.11) with respect to each of the four parameters span $m \bmod \mathcal{J}(E)$. Let

$$
T_{i}(x, y)=E_{r_{i}}\left(u_{1}, u_{2}, P^{*}, 0\right)-E_{r_{i}}\left(u_{1}^{*}, u_{2}^{*}, P^{*}, 0\right)
$$

where $x=u_{1}-u_{1}^{*}, y=u_{2}-u_{2}^{*}$. These are easily computed using (5.1).

$$
\begin{aligned}
& T_{1}(x, y)=\widehat{a} x+\widehat{b} x^{2}+\widehat{c} x^{3}+\widehat{d} x^{4}+\cdots \\
& T_{2}(x, y)=\widehat{a} y+\widehat{b} y^{2}+\widehat{c} y^{3}+\widehat{d} y^{4}+\cdots \\
& T_{3}(x, y)=\widehat{A}(x+y)+\widehat{B}(x+y)^{2}+\widehat{C}(x+y)^{3}+\widehat{D}(x+y)^{4}+\cdots .
\end{aligned}
$$

We first want to show that $T_{1}, T_{2}$, and $T_{3}$ are linearly independent in $m$ mod $\mathcal{J}(E)$. It is more convenient to use $\mathcal{T}_{1}=T_{1}+T_{2}, \mathcal{T}_{2}=T_{1}-T_{2}$ and $\mathcal{T}_{3}=T_{3}$ as unfolding directions. We need to express these functions using $\left(w_{1}, w_{2}\right)$ coordinates. The change of coordinates formula from $(s, t)$ to $\left(w_{1}, w_{2}\right)$ is given in the proof of Theorem 5.3. We solve for $(s, t)$ in terms of $\left(w_{1}, w_{2}\right)$ keeping in mind that our functions are to be expressed as members of $m$ $\bmod \mathcal{J}(E)$. We find $s=w_{2}^{2}-\alpha w_{2}^{4} \bmod \mathcal{J}(E)$ and $t=w_{2}$ whence

$$
\begin{aligned}
& \mathcal{T}_{1}=\left(\widehat{a}+\frac{\sigma^{2} \widehat{b}}{2}\right) w_{2}^{2}+\left(-\alpha \widehat{a}+\frac{\widehat{b}}{2}+\frac{3 \sigma^{2} \widehat{c}}{4}+\frac{\sigma^{4} \widehat{d}}{8}\right) w_{2}^{4} \\
& \mathcal{T}_{2}=\sigma\left\{\widehat{a} w_{2}+\left(\widehat{b}+\frac{\sigma^{2} \widehat{c}}{4}\right) w_{2}^{3}\right\} \\
& \mathcal{T}_{3}=\widehat{A} w_{2}^{2}+(-\alpha \widehat{A}+\widehat{B}) w_{2}^{4}, \quad \bmod \mathcal{J}(E) .
\end{aligned}
$$

At $P^{*}$ these formulas can be simplified using (5.10)-(5.12). The coefficients are to be evaluated at $r_{i}=r_{i}^{*}$ and $u_{i}=u_{i}^{*}$ where we have tended to drop the star superscript in writing the formulas. Namely, from (5.12) we have

$$
\sigma^{2}=-8 B_{2} / A_{3}, \quad B_{2}=\frac{1}{r_{3}^{4}} F^{\prime \prime}\left(u_{3} / r_{3}^{3}\right), \quad A_{3}=\frac{1}{r_{1}^{7}} F^{\prime \prime \prime}(1) .
$$

We obtain $\sigma^{2}=(27 / 2)\left(r_{1}^{7} / r_{3}^{4}\right) F^{\prime \prime}\left(u_{3} / r_{3}^{3}\right)$ as $F^{\prime \prime \prime}(1)=-16 / 27$. However, at $P^{*}$ we can also use (5.17) with $C=1$ giving us $\sigma^{2}=3 r_{1}^{3}$. The formulas for $\widehat{a}, \widehat{b}, \widehat{c}$ involve the rescaled energy function $F(u)$ and its first four derivatives evaluated at $u=1$. The formulas for $\widehat{a}, \widehat{b}$ and $\widehat{c}$ involve the appropriate derivatives of $E$ evaluated at $P^{*}$. We find

$$
\begin{aligned}
& \widehat{a}=E_{u_{1} r_{1}}=-4 / 3 r_{1}^{2} \\
& \widehat{b}=\frac{1}{2} E_{u_{1} u_{1} r_{1}}=8 / 9 r_{1}^{5}
\end{aligned}
$$




$$
\widehat{c}=\frac{1}{6} E_{u_{1} u_{1} u_{1} r_{1}}=-40 / 81 r_{1}^{8} .
$$

This leads to

$$
\mathcal{T}_{2}=\sigma\left\{\left(\frac{-4}{3 r_{1}^{2}}\right) w_{2}+\left(\frac{14}{27 r_{1}^{5}}\right) w_{2}^{3}\right\} \quad \bmod \mathcal{J}(E) .
$$

We observe that $\mathcal{T}_{2}$ is an odd function of $w_{2}$.

Next we establish the linear independence of $\mathcal{T}_{1}, \mathcal{T}_{3}$ both even functions of $w_{2}$. At $P^{*}$ one finds that the coefficient of $w_{2}^{2}$ in the expansion of $\mathcal{T}_{1}$ equals zero while

$$
\widehat{A}=\frac{1}{r_{3}^{2}} F^{\prime}\left(u_{3} / r_{3}^{3}\right)+\frac{3 u_{3}}{r_{3}^{5}} F^{\prime \prime}\left(u_{3} / r_{3}^{3}\right)
$$

is positive because $\left(u_{3} / r_{3}^{3}\right)<1$. Thus we need only to check that the coefficient of $w_{2}^{4}$ in the expansion of $\mathcal{T}_{1}$ is not zero. The formula for $\widehat{d}$ involves the fourth and fifth derivatives of $F(u)$ at $u=1$. With the aid of Lemma 2.1 we can find the fifth derivative. In fact $F^{(5)}(1)=-(2240 / 243)$. This gives us

$$
\widehat{d}=\frac{1}{24} E_{u_{1} u_{1} u_{1} u_{1} r_{1}}=\frac{40}{243 r_{1}^{11}} .
$$

The coefficient of $w_{2}^{4}$ reduces to

$$
\frac{4 \alpha}{3 r_{1}^{2}}-\frac{13}{27 r_{1}^{5}} \cong \frac{.95}{r_{1}^{5}}
$$

where we have used the expression for $\alpha$ given in (5.12) and computed in (5.29). The coefficient of $w_{2}^{4}$ is positive and the functions $\mathcal{T}_{1}, \mathcal{T}_{2}$ and $\mathcal{T}_{3}$ are linearly independent in $m \bmod \mathcal{J}(E)$.

Similarly the $\epsilon$ unfolding direction is computed by

$$
E_{\epsilon}\left(u_{1}, u_{2}, P^{*}, 0\right)-E_{\epsilon}\left(u_{1}^{*}, u_{2}^{*}, P^{*}, 0\right) .
$$

By (6.11) we see that the energy function splits into the sum of three terms with $\epsilon$ present only in the first two. Considering the first term we have by $(6.10)$

$$
E_{\epsilon}\left(u_{1}, r_{1}^{*}, 0\right)=\frac{1}{\pi} P\left(u_{1}, r_{1}^{*}, 0\right)
$$

where $P\left(u_{1}, r_{1}, 0\right)$ is the gravitational potential energy of a spherical cap with volume $v=(2 \pi / 3)\left(u_{1} / r_{1}^{3}\right)$ and boundary the circle of radius $r$ in the horizontal plane. Go back to the formulation of Section 2 where we computed area and volume as function of $(r, t)$ where $(0, t)$ located the center of the generating circle. A direct calculation gives

$$
\iiint z d x d y d z=\frac{\pi}{12}\left(t+\sqrt{r^{2}+t^{2}}\right)\left(3 \sqrt{r^{2}+t^{2}}-t\right)
$$


where integration is over the solid region bounded by the spherical cap and the disk of radius $r$. We must expand $P$ as a function of volume about the value $u_{1}=u_{1}^{*}$ with $u_{1}^{*}=r_{1}^{* 3}$ which is the hemisphere configuration. After some calculation we find after setting $x=u_{1}-u_{1}^{*}$

$$
\begin{aligned}
\pi \cdot T_{4}(x, y) & =P\left(u_{1}, r_{1}^{*}, 0\right)-P\left(u_{1}^{*}, r_{1}^{*}, 0\right) \\
& =\left(4 r_{1}^{*} / 9\right) x+\left(4 / 27 r_{1}^{* 2}\right) x^{2}-\left(8 / 243 r_{1}^{* 5}\right) x^{3}+\cdots .
\end{aligned}
$$

Using (6.11) the total derivative with respect to $\epsilon$ of the energy at $\epsilon=0$ is just $\mathcal{T}_{4}=T_{4}(x)-T_{4}(y)$, giving us another unfolding which will be odd in the variable $w_{2}$ after changing coordinates from $(x, y)$ to $\left(w_{1}, w_{2}\right)$ as before. As in the calculation of $\mathcal{T}_{2}$ we now use (6.14) along with the fact that at $P^{*}$, $\sigma^{2}=3 r_{1}^{* 3}$ giving us

$$
\pi \mathcal{T}_{4}=\left(\sigma / 81 r_{1}^{* 2}\right)\left\{\left(36 r_{1}^{* 3}\right) w_{2}+5 w_{2}^{3}\right\} \quad \bmod \mathcal{J}(E) .
$$

Comparing (6.15) with (6.21) we see that $\mathcal{T}_{2}, \mathcal{T}_{4}$ are linearly independent. The unfolding is universal.

Addendum to Theorem 6.2. Let $\widehat{P}=\left(\widehat{r}_{1}, \widehat{r}_{2}, \widehat{r}_{3}\right)$ be a point on the cusp curve $[A]$ other than butterfly point $P^{*}$. In this case the parameters $r_{1}, r_{2}$ are unfolding directions and $E\left(u_{1}, u_{2}, r_{1}, r_{2}, \widehat{r}_{3}\right)$ is a universal unfolding.

Proof. From Theorem 5.3 we know that at points $\widehat{P}$ on the cusp curve $[A]$ other than $P^{*}$ the 4 -jet of the energy $E$ (up to a constant) has the form $j^{4}(E)=w_{1}^{2}+D w_{2}^{4}$ with $D=C-1 \neq 0$ where $C$ is given by (5.8). This gives the cusp catastrophe with $\operatorname{det}(E)=4$ and $\operatorname{cod}(E)=2$. In fact the Jacobi ideal is $\mathcal{J}(E)=\left\langle w_{1}, w_{2}^{3}\right\rangle_{\mathcal{E}}$ so that $m \bmod \mathcal{J}(E)$ is spanned by $w_{2}, w_{2}^{2}$. Following the discussion in the proof of Theorem 6.2 setting $\epsilon=0$ there and using (6.14) we need to show that

$$
\begin{aligned}
& \mathcal{T}_{1}=\left(\widehat{a}+\frac{\sigma^{2} \widehat{b}}{2}\right) w_{2}^{2} \\
& \mathcal{T}_{2}=\sigma \widehat{a} w_{2} \quad \bmod \mathcal{J}(E)
\end{aligned}
$$

span $m \bmod \mathcal{J}(E)$. Following the calculations given there we find $\sigma^{2}=$ $3 \widehat{r}_{1}^{3} C>0, \widehat{a}=-4 / 3 \widehat{r}_{1}^{2}$ and $\widehat{b}=8 / 9 \widehat{r}_{1}^{5}$ which leads to

$$
\widehat{a}+\frac{\sigma^{2} \widehat{b}}{2}=\frac{4}{3 \widehat{r}_{1}^{2}}(C-1) \neq 0 \quad \text { for } \widehat{P} \neq P^{*} .
$$

The function $\mathcal{T}_{1}, \mathcal{T}_{2}$ are linearly independent in $m \bmod \mathcal{J}(E)$ and the unfolding $E\left(u_{1}, u_{2}, r_{1}, r_{2}, \widehat{r}_{3}\right)$ is universal.

We now show that introducing surface tension as a parameter also generates a universal unfolding at the butterfly point. 
Theorem 6.3. Let $P^{*}$ be the butterfly point on the cusp curve $[A]$. Let $E$ be the energy function obtained by introducing surface tension

$$
E\left(u_{1}, u_{2}, r_{1}, r_{2}, r_{3}, c_{1}, c_{2}, c_{3}\right)=\sum_{i=1}^{3} c_{i} r_{i}^{2} F\left(u_{i} / r_{i}^{3}\right) .
$$

Consider the perturbation at $P^{*}=\left(r_{1}^{*}, r_{2}^{*}, r_{3}^{*}\right)$ and at $c_{1}=c_{2}=c_{3}=1$ obtained by increasing $c_{1}$, decreasing $c_{2}$ and keeping $c_{3}$ fixed so that $c_{1}=c$, $c_{1}+c_{2}=2$ and $c_{3}=1$ with $c$ as parameter. This generates a fourth universal unfolding parameter.

Proof. We follow the calculations used in the proof of Theorem 6.2. First, differentiate the energy function (6.23) in the direction $c_{1}$ to obtain

$$
E_{c_{1}}\left(u_{1}, u_{2}, P^{*}, 1,1,1\right)=r_{1}^{* 2} F\left(u_{1} / r_{1}^{* 3}\right) .
$$

The unfolding parameter $c_{1}$ leads to the unfolding direction

$$
T_{4}(x, y)=E_{c_{1}}\left(u_{1}, u_{2}, P^{*}, 1,1,1\right)-E_{c_{1}}\left(u_{1}^{*}, u_{2}^{*}, P^{*}, 1,1,1\right)
$$

where $x=u_{1}-u_{1}^{*}, y=u_{2}-u_{2}^{*}$ leading to

$$
T_{4}(x, y)=\widehat{a} x+\widehat{b} x^{2}+\widehat{c} x^{3}+\widehat{d} x^{4}+\cdots
$$

similar to (6.13). In the present instance the coefficients $\widehat{a}, \widehat{b}, \widehat{c}, \widehat{d}$ are easily computed. We find $\widehat{a}=4 / 3 r_{1}^{*}, \widehat{b}=0, \widehat{c}=8 / 81 r_{1}^{* 7}, \widehat{d}=8 / 81 r_{1}^{* 10}$. Differentiation of the energy functional with respect to $c_{2}$ gives us a similar function $T_{4}^{\prime}$ where $x=u_{1}-u_{1}^{*}$ is replaced by $y=u_{2}-u_{2}^{*}$. We have selected $c=c_{1}$, $c_{2}=2-c, c_{3}=1$ so that the unfolding direction is $\mathcal{T}_{4}=T_{4}-T_{4}^{\prime}$. This is written in $w_{1}, w_{2}$ coordinates in (6.14). We obtain

$$
\mathcal{T}_{4}=\sigma\left\{\left(4 / 3 r_{1}^{*}\right) w_{2}+\left(2 / 27 r_{1}^{* 4}\right) w_{2}^{3}\right\} \quad \bmod \mathcal{J}(E) .
$$

Upon comparing this with the expression for $\mathcal{T}_{2}$ given in $(6.15)$ we see that $\mathcal{T}_{2}, \mathcal{T}_{4}$ are linearly independent. We have a universal unfolding.

Note. It might seem that introducing surface tension as a parameter is just a rescaling device and so should be equivalent to our original problem. However, this is not the case as any rescaling would also introduce weighted sums of the total volume.

Finally, we now consider unfoldings induced by perturbations of the boundary circles and will show that such variations fail to lead to a universal unfolding at $P^{*}$. For a given volume $v$ let $\Sigma_{0}$ be the spherical cap in the upper half space with boundary the unit circle $C_{0}$ in the $x-y$ plane which along with the unit disk $M_{0}$ encloses a solid region $T_{0}$ whose volume is $v$. We define a perturbation of the boundary curve $C_{0}$ as follows. 
Definition 6.2. Let $f(\theta), g(\theta)$ be smooth $C^{\infty}$-functions with period $2 \pi$. Define a one-parameter family of curves $C_{\epsilon}$ by

$$
x_{\epsilon}(\theta)=(1+\epsilon f(\theta)) b(\theta)+\epsilon g(\theta) k
$$

where $b(\theta)=\langle\cos \theta, \sin \theta, 0\rangle$ is the position vector for $C_{0}$ and $k$ is the unit vector in the direction of the $z$-axis. We observe that $C_{\epsilon}$ is described by a normal perturbation of $C_{0}$. For convenience we make the perturbation linear in $\epsilon$. Furthermore suppose that there are $C^{\infty}$-functions $L(\theta), \beta(\theta)$ of period $2 \pi$ such that for each $\theta, f=L \cos \beta$ and $g=L \sin \beta$. Note that the family of curves collectively form a parametric surface surrounding $C_{0}$.

Given a family of curves $C_{\epsilon}$ as described with $C_{0}$ the unit circle we consider a one-parameter family of pair of surfaces $\Sigma_{\epsilon}, M_{\epsilon}$ where $\Sigma_{\epsilon}$ is a smoothly varying family of surfaces with $\Sigma_{0}$ the initial spherical cap such that the boundary of $\Sigma_{\epsilon}$ is the perturbed curve $C_{\epsilon}$. Suppose the spherical cap is part of the sphere $S_{0}$ of radius $R$ whose center lies on the $z$-axis. The surface $\Sigma_{0}$ along with the unit disk enclose a volume $v$ and we let $\alpha$ denote the interior angle of contact of $\Sigma_{0}$ with $M_{0}$ along the unit circle $C_{0}$. Let $p$ denote a point on $S_{0}$ and $\xi=\xi(p)$ the unit normal vector to $S_{0}$. We assume that $\Sigma_{\epsilon}$ can be represented by a normal graph over a region on $S_{0}$ whose boundary is the normal projection of $C_{\epsilon}$ on $S_{0}$.

$$
x(p, \epsilon)=p+\phi(p, \epsilon) \xi(p)=p+\epsilon N(p) \xi(p)+o(\epsilon) .
$$

Here $\phi(p, \epsilon), N(p)$ are $C^{\infty}$-functions on a region containing the closure of the domain describing the surface $\Sigma_{\epsilon}$. The function $N(p)=\phi_{\epsilon}(p, 0)$ is the "initial speed" of the perturbation with $N \xi$ the initial vector field of the perturbation.

For small $\epsilon$ the curves $C_{\epsilon}$ project onto a convex curve $\Gamma_{\epsilon}$ in the plane. It follows that there is a uniquely determined minimal surface $M_{\epsilon}$ with boundary $C_{\epsilon}$ which can be represented in non-parametric form.

$$
z=f(x, y, \epsilon)=\epsilon \widetilde{N}(x, y)+o(\epsilon)
$$

where $\tilde{N}(x, y)=f_{\epsilon}(x, y, 0)$. The functions $f(x, y, 0)$ are solutions to the minimal surface equation and can be extended in a smooth manner to be $C^{\infty}$-functions in some region containing $M_{0} \times\left[-\epsilon_{0}, \epsilon_{0}\right]$. Since $f(x, y, 0)=0$ it follows that $\tilde{N}(x, y)$ is a harmonic function on the unit disk.

We assume that the pair $\Sigma_{\epsilon}, M_{\epsilon}$ together bound a solid region $T_{\epsilon}$ whose volume is the given constant $v$. We have that $\Sigma_{0}$ is the spherical cap and imagine that each $\Sigma_{\epsilon}$ is also a cmc surface but for our calculations this assumption is not needed. We have the following result.

Theorem 6.4. Let $C_{\epsilon}$ be the smooth one-parameter family of boundary curves as given in Definition 6.2 and suppose $\Sigma_{\epsilon}, M_{\epsilon}$ is a one-parameter family with common boundary $C_{\epsilon}$ as just described with $\Sigma_{0}$ the spherical 
cap, $M_{0}$ the unit disk and such that the pair $\Sigma_{\epsilon}, M_{\epsilon}$ together enclose volume $v$. Let $A(v, \epsilon)$ be the area of the surface $\Sigma_{\epsilon}$. We have the following formula for the derivative of this area function with respect to $\epsilon$ at $\epsilon=0$ :

$$
A_{\epsilon}(v, 0)=\cos \alpha \int_{0}^{2 \pi} f(\theta) d \theta
$$

where $f(\theta), g(\theta)$ are those $C^{\infty}$-functions describing the curves $C_{\epsilon}$ in Definition 6.2 .

Proof. We have $\alpha$ as the interior angle of contact of $\Sigma_{0}$ with $M_{0}$. Let $\gamma=$ $\gamma(\theta)$ be the interior agle of contact of $\Sigma_{0}$ with the surface determined by the $C_{\epsilon}$. One sees that $\gamma(\theta)=\alpha+\beta(\theta)$ where $\beta(\theta)$ is the angle of inclination of the straight line determined by the normal slice to $C_{0}$ of this surface as in Definition 6.2. We have the following facts.

a)

$$
A_{\epsilon}(v, 0) \equiv \delta A(N)=\iint_{\Sigma_{0}}-2 H N d A+\int_{C_{0}} N \cot \gamma d s .
$$

This is the classic formula for the first variation of area $[\mathbf{7}$, p. 9] or $[\mathbf{1 1}$, p. 457]. Here the mean curvature $H$ of the spherical cap is negative with $\sin \alpha=1 / R=-H$. Also, since $f=L \cos \beta$ one sees that along $C_{0}$ we have $N=L \sin \gamma$ so that $N \cot \gamma=L \cos \gamma$.

b) Since the volume $v$ remains constant with $\epsilon$ we find

$$
0=\iint_{\Sigma_{0}} N d A-\iint_{M_{0}} \tilde{N} d A
$$

(see either $[\mathbf{7}, \mathbf{1 1}])$.

c) Since $\widetilde{N}$ is harmonic on $M_{0}$ we have by the mean value property for harmonic functions

$$
\iint_{M_{0}} \tilde{N} d A=\frac{1}{2} \int_{C_{0}} \tilde{N} d s
$$

d) Since $\Sigma_{\epsilon}$ and $M_{\epsilon}$ both span $C_{\epsilon}$ we find (referring to Definition 6.2) that on $C_{0}$

$$
L=N c s c \gamma=\tilde{N} \csc \beta .
$$

Now use the information above to express $\delta A(N)$ as a line integral over $C_{0}$. We find

$$
\begin{aligned}
\delta A(N) & =\int_{C_{0}}(N c s c \gamma)(\cos \gamma+\sin \alpha \sin \beta) d s \\
& =\int_{C_{0}}(L)(\cos \alpha \cos \beta) d s .
\end{aligned}
$$

Finally note that $L \cos \beta=f$ and upon switching to integration with respect to $\theta$ we obtain (6.29). 
Corollary. Suppose we have an unfolding of the triple pipe configuration determined by a perturbation of the boundary curves $C_{\epsilon}$ as given by Definition 6.2. An equivalent unfolding is given by a simple expansion of the boundary circles $C_{\epsilon}^{\prime}: x(\epsilon, \theta)=\left(1+\epsilon f_{o}\right) b(\theta)$ where

$$
2 \pi f_{o}=\int_{0}^{2 \pi} f(\theta) d \theta .
$$
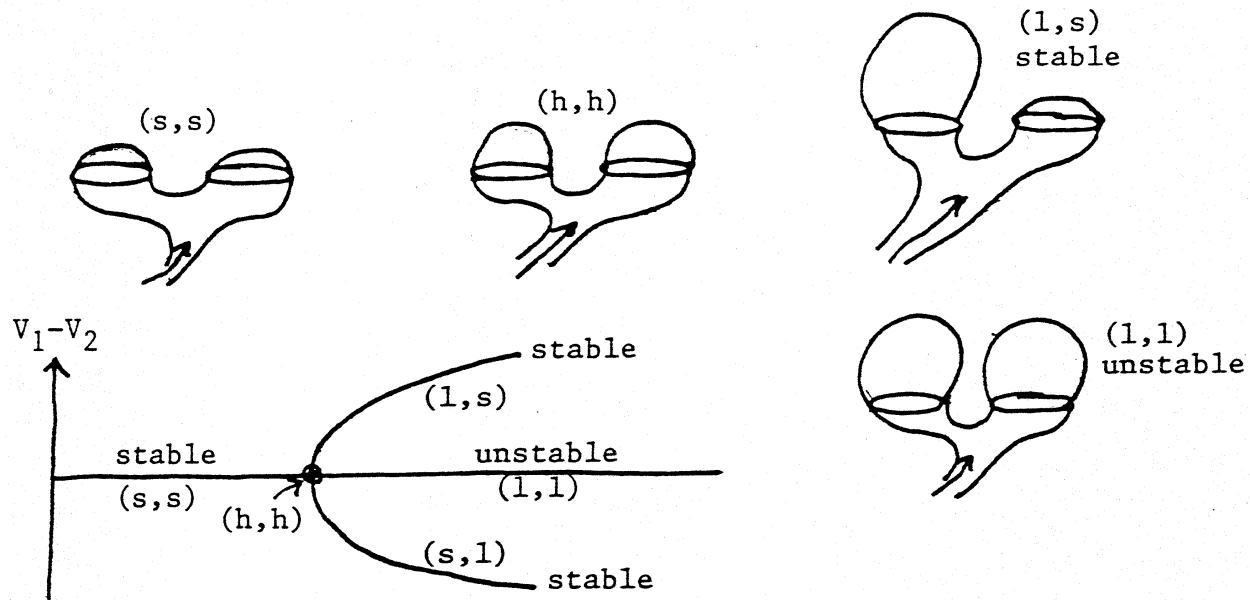

The Two Bubble Bifurcation.
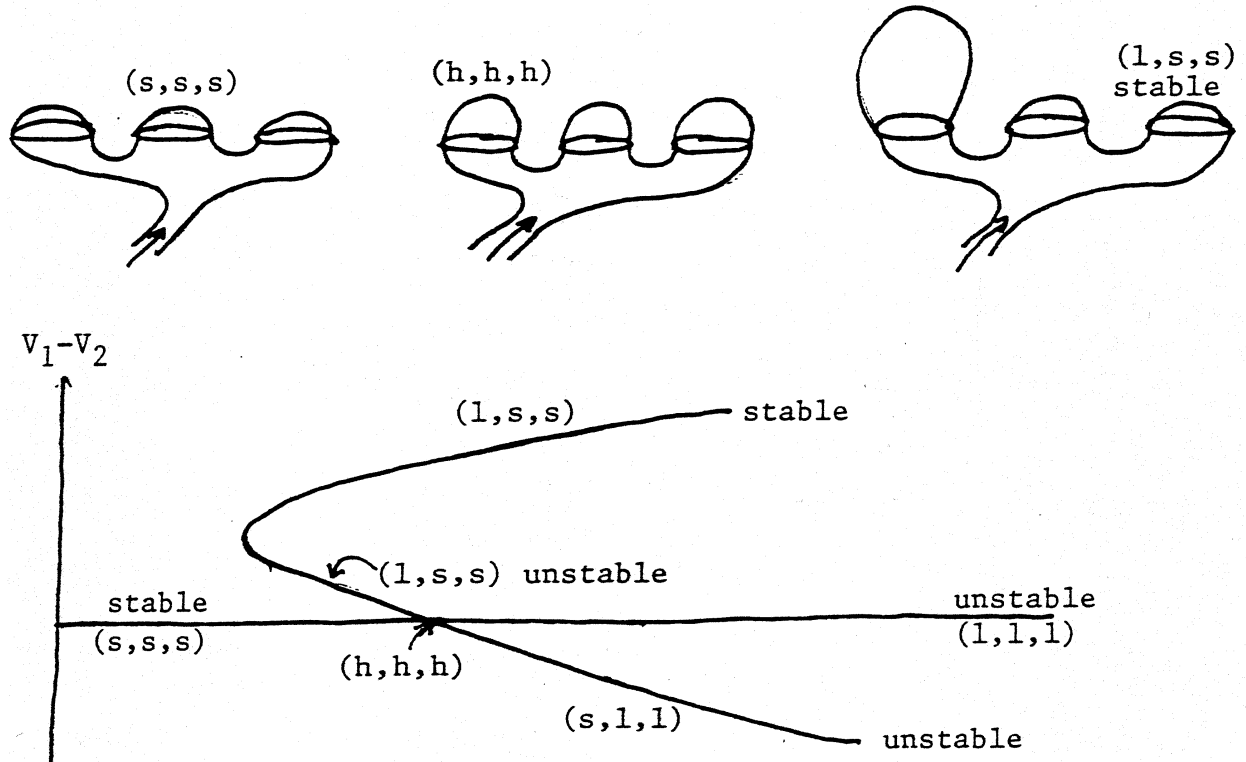

The Three Bubble Catastrophe.

Figure 1. 

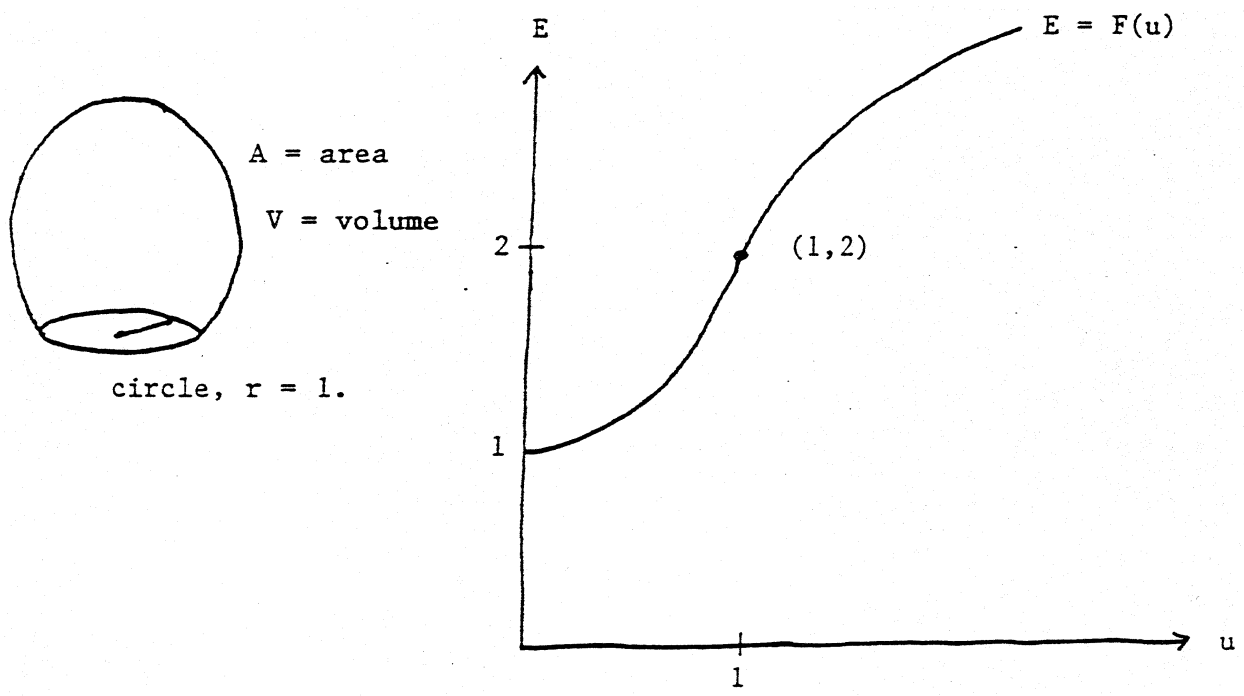

$A=$ Area of spherical cap.

$V=$ Volume of cap.

$E=\frac{1}{\pi} A\left(\frac{2 \pi}{3} u\right)=$ the scaled energy $=F(u)$.

$u=0 \Rightarrow$ Flat Disk $\Rightarrow A=\pi$ so $E=1$.

$u=1 \Rightarrow$ hemisphere $\Rightarrow A=2 \pi$ so $E=2$.

1) $F(0)=1, F(1)=2$.

2) $F^{\prime}(u)>0$ when $u>0$.

3) $F^{\prime \prime}(u)=\left\{\begin{array}{ll}>0, & 0 \leq u<1 \\ =0, & u=1 \\ <0, & u>1\end{array}\right\}$.

4) $F^{\prime \prime \prime}(1)=-\gamma=-16 / 27<0$.

5) $F^{(4)}(1)=\delta=64 / 27>0$.

Figure 2. The Energy Function. 

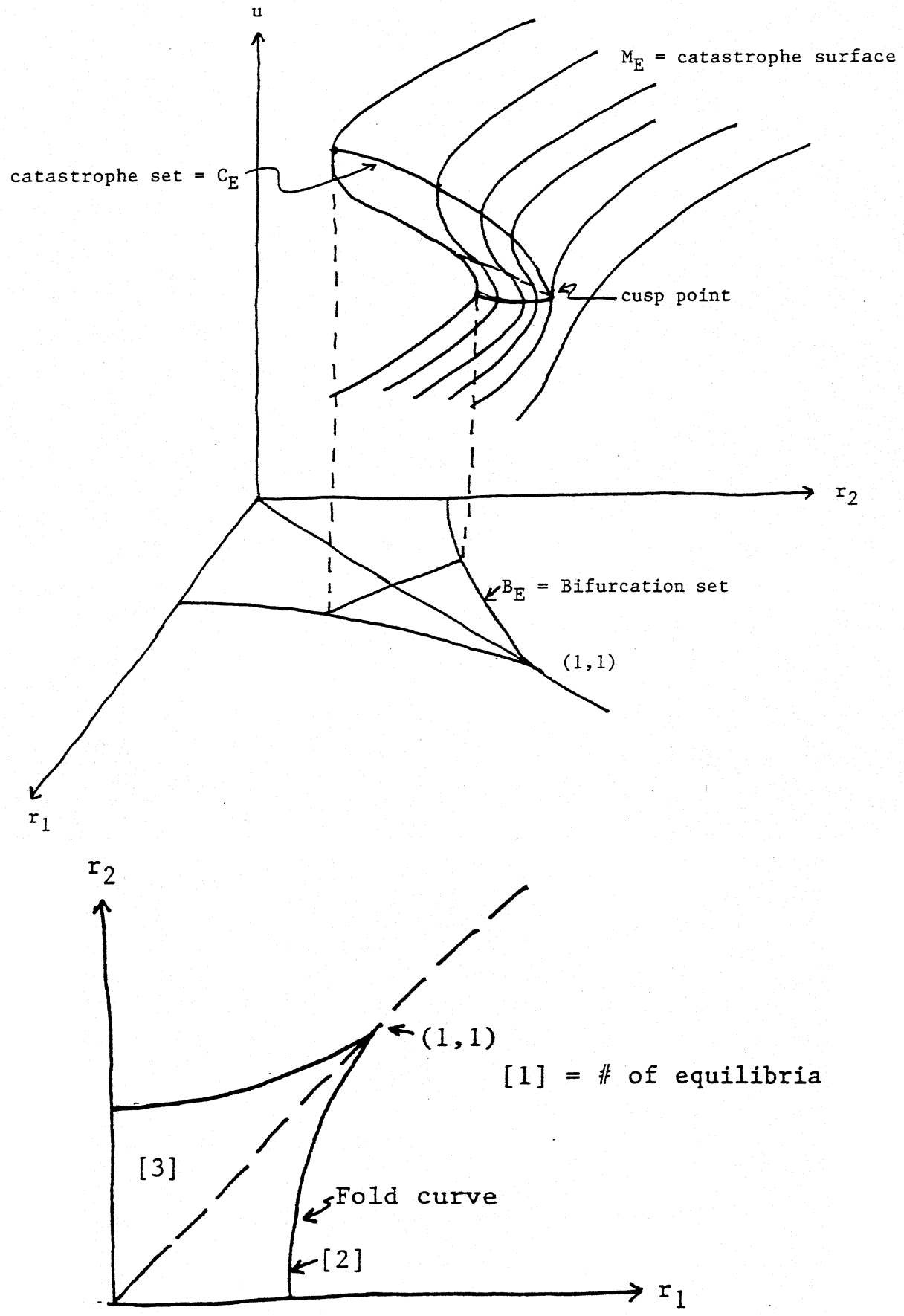

Figure 3. The Double Bubble Cusp Catastrophe. 


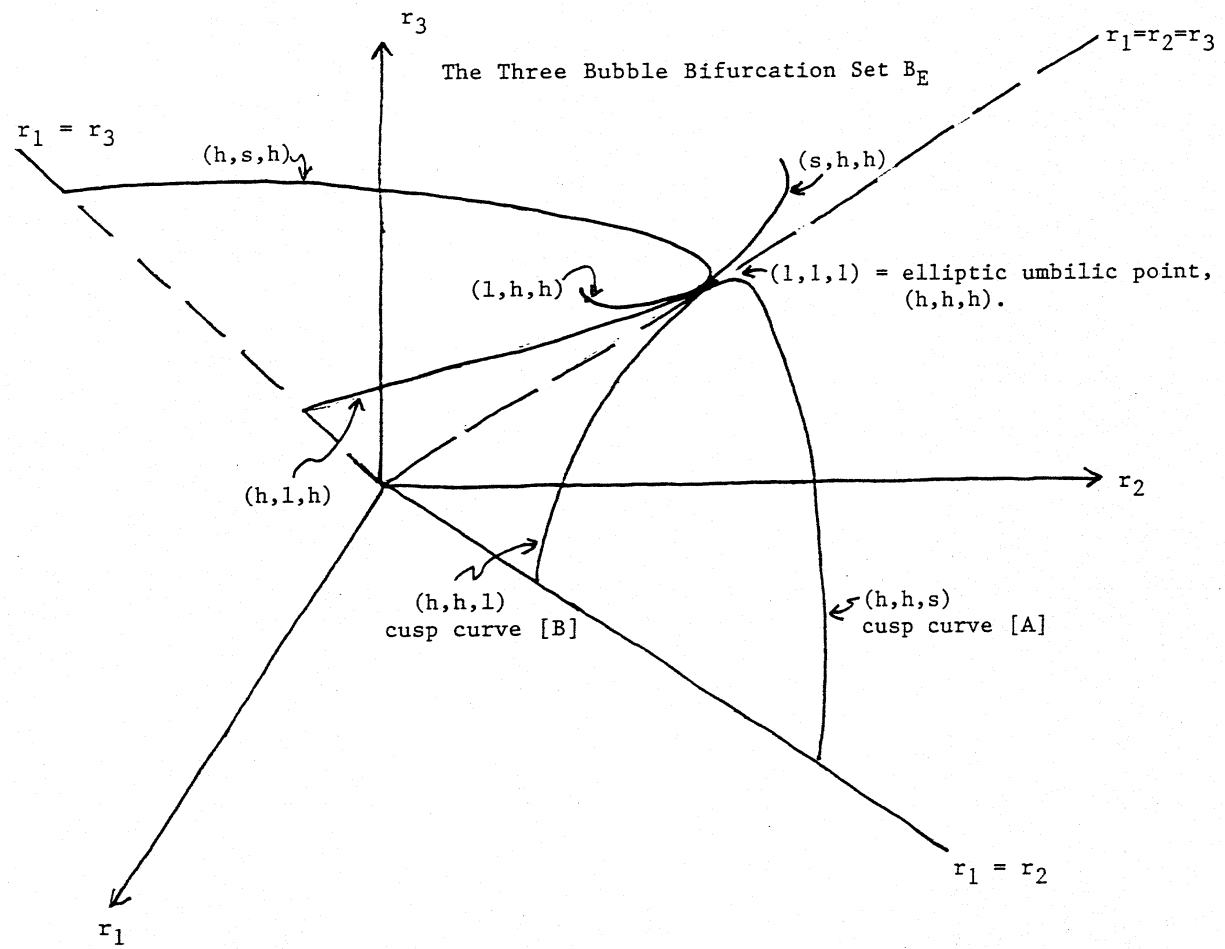

Three Cusp Curves emanate from an Elliptic Umbilic Point. They are connected by Fold Surfaces.

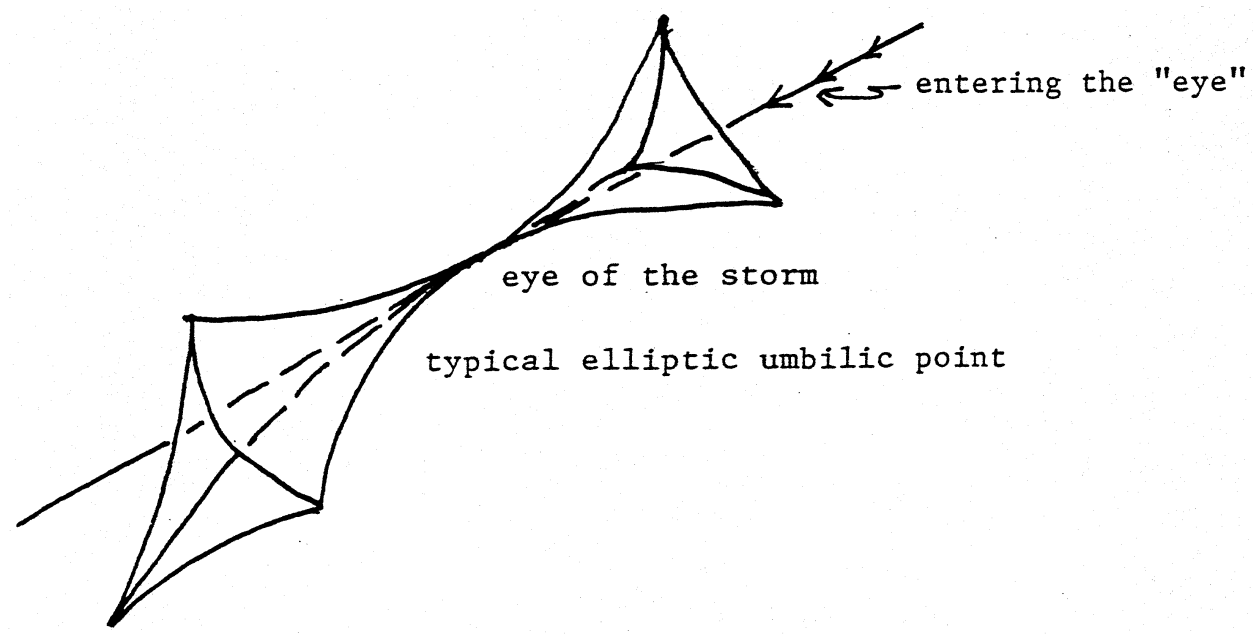

Figure 4. 


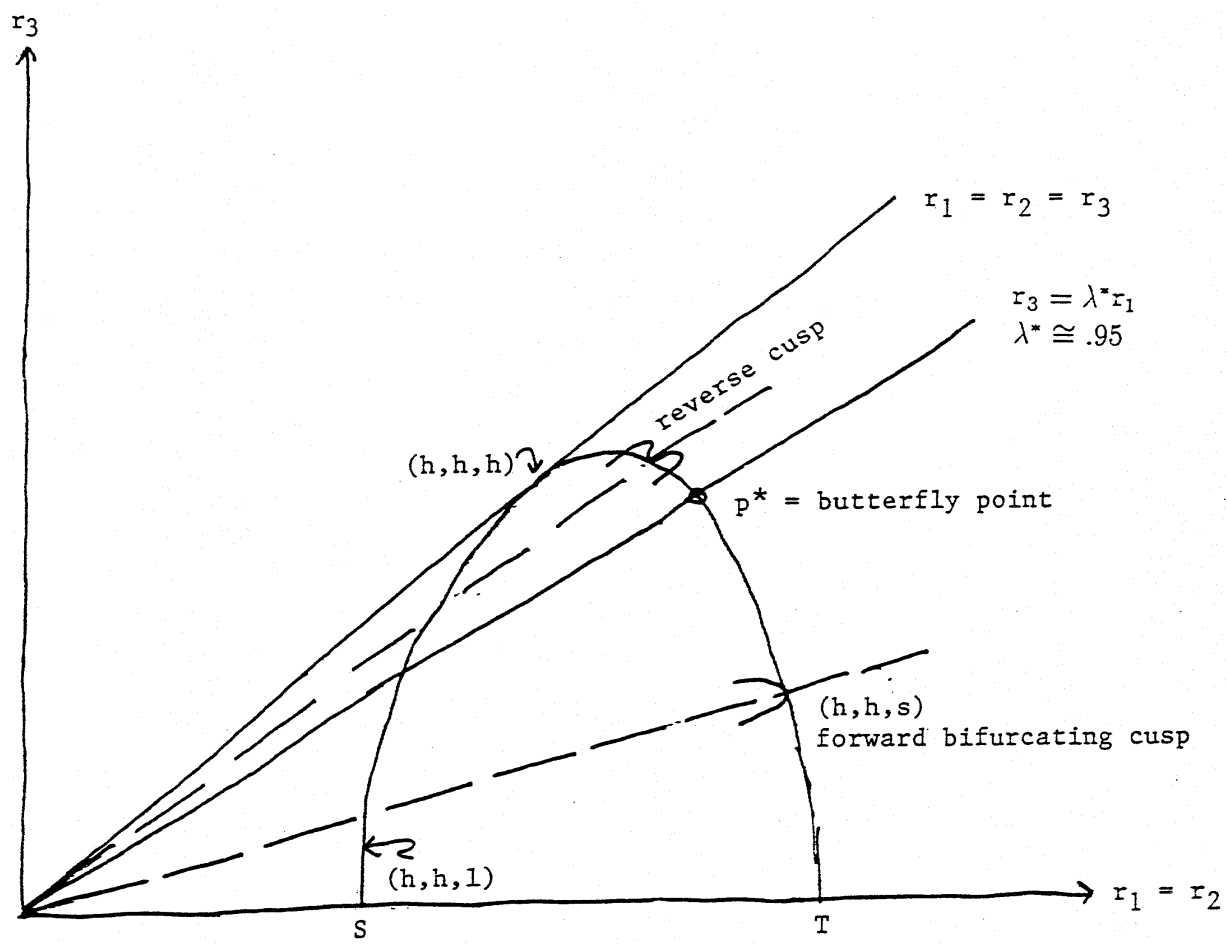

Figure 5. The cusp curve when $r_{1}=r_{2}=r_{3}$.

At $S$ we have $\square+\square+\bigcirc$. Total volume $=3\left(\frac{2 \pi}{3}\right)=4\left(\frac{2 \pi}{3}\right) r_{1}^{3} \Longrightarrow r_{1}^{3}=\frac{3}{4}$ or $r_{1}=.908$.

At $T$ we have $\square+\square+\cdots$. Total volume $=3\left(\frac{2 \pi}{3}\right)=2\left(\frac{2 \pi}{3}\right) r_{1}^{3} \Longrightarrow r_{1}^{3}=\frac{3}{2}$ or $r_{1}=1.1447$.

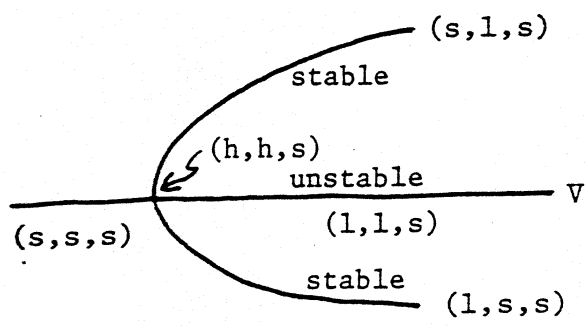

Bifurcation at cusp point $(h, h, s)$ when $r_{3}<\lambda^{*} r_{1}$.

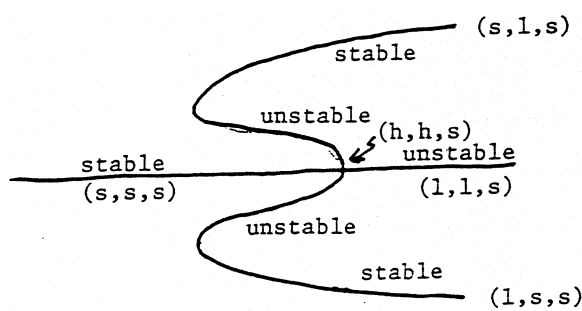

Bifurcation at cusp point $(h, h, s)$ when $r_{3}>\lambda^{*} r_{1}$.

Figure 6. 


\section{References}

[1] A.W. Adamson, Physical Chemistry of Surfaces, 2nd edition, Interscience, NY, 1967.

[2] M.J. Beeson and A.J. Tromba, The cusp catastrophe of Thom in the bifurcation of minimal surfaces, Manuscripta Math., 46 (1984), 273-308.

[3] C.V. Boys, Soap Bubbles and the Forces Which Mould Them, Society for Promoting Christian Knowledge, London, 1902; Dover reprint, NY, 1959.

[4] J. Büch, Bifurcation von Minimalflächen und elementare Katastrphen, Manuscripta Math., 55 (1986), 269-306.

[5] D. Castrigiano and S. Hayes, Catastrophe Theory, Addison Wesley, 1993.

[6] F. Duzaar and K. Steffen, Area minimizing hypersurfaces with prescribed volume and boundary, Math. Zeitschrift, 209 (1992), 581-618.

[7] R. Finn, Equilibrium Capillary Surfaces, Springer, 1986.

[8] A.T. Fomenko, The Plateau Problem, Part I, Gordon and Breach, Amsterdam, 1990.

[9] J.C.C. Nitsche, Contours bounding at least three solutions of Plateau's problem, Arch. Rat. Mech. Anal., 30 (1968), 1-11.

[10] T. Poston and I. Stewart, Catastrophe Theory and Its Applications, Pitman, London, 1978.

[11] H.C. Wente, The stability of the axially symmetric pendant drop, Pacific J. Math., $8 \mathbf{8}$ (1980), 421-470.

Received January 3, 1996 and revised June 2, 1998. Work on this paper was done while the author was a guest of the University of Bonn (SFB 256) and the Max Planc Institute, Bonn, Germany.

UNIVERSity OF TOLEDO

Toledo, Ohio 43606

E-mail address: hwente@math.utoledo.edu 